MATHEMATICS OF COMPUTATION

Volume 68, Number 226, April 1999, Pages 461-485

S 0025-5718(99)01063-7

\title{
NUMERICAL SOLUTION OF A FAST DIFFUSION EQUATION
}

\author{
MARIE-NOELLE LE ROUX AND PAUL-EMILE MAINGE
}

\begin{abstract}
In this paper, the authors consider the first boundary value problem for the nonlinear reaction diffusion equation: $u_{t}-\Delta u^{m}=\alpha u^{p_{1}}$ in $\Omega$, a smooth bounded domain in $\mathbb{R}^{d}(d \geq 1)$ with the zero lateral boundary condition and with a positive initial condition, $m \in] 0,1[$ (fast diffusion problem), $\alpha \geq 0$ and $p_{1} \geq m$. Sufficient conditions on the initial data are obtained for the solution to vanish or become infinite in a finite time. A scheme for the discretization in time of this problem is proposed. The numerical scheme preserves the essential properties of the initial problem; namely existence of an extinction or a blow-up time, for which estimates have been obtained. The convergence of the method is also proved.
\end{abstract}

\section{INTRODUCTION}

In this paper, a numerical scheme is proposed to solve the reaction diffusion problem: find a nonnegative function $u$ defined on $\Omega$, a smooth domain $\mathbb{R}^{d}(d \geq 1)$ and such that

$$
\begin{array}{cccc}
u_{t}-\Delta u^{m} & = & \alpha u^{p_{1}} & x \in \Omega, t>0 \\
u(t, x) & = & 0 & x \in \partial \Omega, t \geq 0 \\
u(0, x) & = & u_{0}(x)>0 & x \in \Omega,
\end{array}
$$

where $m \in] 0,1\left[\right.$ (fast diffusion problem), $\alpha>0$ and $p_{1} \geq m$.

This problem and analogous problems have been studied from a theoretical point of view by several authors: Aronson-Crandall-Peletier [2], Berryman-Holland [3], Friedman-Lacey [4], Friedman-McLeod [5], [6], Levine-Sacks [13], Sabinina [15], and Sacks [16], [17], [18]. M.-N. Le Roux has proposed a numerical method in [8] and [9] to compute the solution of a similar problem (1.1) with $m>1$ (slow diffusion problem).

In the case $\alpha=0$, there exists an extinction time $T^{*}$ such that the problem (1.1) has a unique classical solution, positive on $\Omega \times\left[0, T^{*}\left[\right.\right.$ and null for $t \geq T^{*}$ (see [3], [15]). Concerning this last case, a semidiscretization in time is proposed in [10] by M.-N. Le Roux, for which the numerical solution has the same properties as the exact solution, so it allows the calculation of a numerical extinction time.

In the case $\alpha>0$, according to the values of $p_{1}$, the solution of (1.1) may vanish or blow up in a finite time:

- For $p_{1} \in[m, 1]$, the solution of (1.1) cannot blow up, but it may vanish in some finite time;

Received by the editor August 13, 1996 and, in revised form, May 5, 1997.

1991 Mathematics Subject Classification. Primary 35K55, 35K57, 65M60.

Key words and phrases. Reaction diffusion equations, parabolic problems.

(C)1999 American Mathematical Society 
- For $p_{1}>1$, it is possible for the solution to vanish or blow up in some finite time according to the initial data.

Here, we study the semidiscretization in time of the problem (1.1): we propose a scheme whose solution has the same properties as the solution of the theoretical problem, in particular extinction or blow up in a finite time. A complete discretization of (1.1) using a $P_{1}$-finite element method has been studied in [14]; the results obtained are the same as for the semidiscretization in time and the proofs are analogous, so, we shall not develop this point. Further, numerical results concerning this problem or similar problems may be found in [12] or [14].

An outline of this paper follows.

In $\S 2$, we recall some theoretical results and set up sufficient conditions on the initial data for the solution to vanish or blow up in a finite time.

In $\S 3$, we define a numerical scheme for the semidiscretization in time of (1.1) and we prove the existence of the numerical solution. An iterative method to solve the nonlinear equation obtained at each time step is proposed and its convergence is proved.

In $\S 4$, we study the behavior of the numerical solution; it has similar properties as the exact solution.

In $\S 5$, we prove the convergence of the numerical method.

\section{Asymptotic Behavior of the SOLUtion}

By using the variable $v=u^{m}$, it is more convenient to work with the transformed equation

$$
\begin{aligned}
& p v^{p-1} v_{t}+A v=\alpha v^{r}, \quad t>0, \\
& v(0) \quad=v_{0}=u_{0}^{m}>0,
\end{aligned}
$$

where $p=\frac{1}{m}, r=\frac{p_{1}}{m}$; so $p>1$ and $r \geq 1$. A denotes the operator $-\Delta$ of domain $D(A)=H_{0}^{1}(\Omega) \cap H^{2}(\Omega)$.

This problem has a unique solution at least on a bounded interval ([16]).

We suppose that $p$ satisfies the following hypothesis:

$$
p>1 \text { if } d \leq 2 ; 1<p<\frac{d+2}{d-2} \text { if } d>2, \quad \quad\left(H_{1}\right)
$$

which assures continuous and compact embedding of $H_{0}^{1}(\Omega)$ in $L^{p+1}(\Omega)$. Then, we denote

$$
C(\Omega)=\operatorname{Inf}_{\varphi \in H_{0}^{1}(\Omega), \varphi \neq 0} \frac{1}{\|\varphi\|_{p+1}^{2}} \int_{\Omega}|\nabla \varphi|^{2} d x
$$

We suppose also that the initial condition is in $H_{0}^{1}(\Omega) \cap C^{\epsilon}(\bar{\Omega}), \epsilon>0$.

For $s \geq 1$, we denote by $\|\cdot\|_{s}$ the natural norm in $L^{s}(\Omega)$ and by $\|\cdot\|_{\infty}$ the one of $L^{\infty}(\Omega)$.

Lemma 2.1. If $r \leq p$, the solution $v$ of (2.1) exists for all $t \geq 0$ and satisfies

$$
\begin{gathered}
\|v(t)\|_{\infty}^{p-r} \leq\left\|v_{0}\right\|_{\infty}^{p-r}+\alpha \frac{p-r}{p} t, \text { if } r<p, \\
\|v(t)\|_{\infty} \leq\left\|v_{0}\right\|_{\infty} \exp \left(\frac{\alpha}{p} t\right), \text { if } r=p .
\end{gathered}
$$


If $r>p$, the solution exists at least on the interval $\left[0, T_{1}\right]$, where

$$
T_{1}=\frac{1}{\alpha} \frac{p}{r-p} \frac{1}{\left\|v_{0}\right\|_{\infty}^{r-p}}
$$

and satisfies

$$
\|v(t)\|_{\infty}^{r-p} \leq\left\|v_{0}\right\|_{\infty}^{r-p} \frac{T_{1}}{T_{1}-t} .
$$

Proof. In the same way as Sacks in [17], we obtain that the solution of (2.1) is bounded by the solution $w$ of the ordinary differential equation

$$
\begin{array}{ccc}
p w^{p-1}(t) w^{\prime}(t) & = & \alpha w^{r}(t), \quad t>0, \\
w(0) & = & \left\|v_{0}\right\|_{\infty} .
\end{array}
$$

The solution $w$ is written $w(t)=\left\|v_{0}\right\|_{\infty}\left(1+\alpha \frac{p-r}{p}\left\|v_{0}\right\|_{\infty}^{r-p} t\right)^{\frac{1}{p-r}}$ if $r \neq p$ and $w(t)=$ $\left\|v_{0}\right\|_{\infty} \exp \left(\frac{\alpha}{p} t\right)$ if $r=p$.

So, it is defined for all $t \geq 0$ if $r \leq p$ and for $t<T_{1}$ if $r>p$ and we deduce the estimates (2.6) and (2.7).

We introduce the Lyapunov functional $J^{*}$ defined by

$$
J^{*}(\varphi)=\frac{1}{2} \int_{\Omega}|\nabla \varphi|^{2} d x-\frac{\alpha}{r+1} \int_{\Omega} \varphi^{r+1} d x \quad \forall \varphi \in H_{0}^{1}(\Omega) \cap L^{r+1}(\Omega) .
$$

Lemma 2.2. The mapping $t \longmapsto J^{*}(v(t))$ is decreasing.

Proof. By multiplying the first equation of (2.1) by $v_{t}$ and integrating on $\Omega$, we obtain

$$
p \int_{\Omega} v^{p-1}\left(v_{t}\right)^{2} d x+\int_{\Omega} \nabla v \nabla v_{t} d x=\alpha \int_{\Omega} v^{r} v_{t} d x
$$

this equality may also be written as $\frac{d}{d t} J^{*}(v(t))=-p \int_{\Omega} v^{p-1}\left(v_{t}\right)^{2} d x$, so the derivative in time of $J^{*}(v)$ is negative, which proves the result.

Lemma 2.3. For $t \geq 0$, we have the inequality

$$
\left\|v_{0}\right\|_{p+1}^{p+1}-2 \frac{p+1}{p} J^{*}\left(v_{0}\right) t \leq\|v(t)\|_{p+1}^{p+1} .
$$

Proof. By multiplying the first equation of (2.1) by $v$ and integrating on $\Omega$, we obtain

$$
p \int_{\Omega} v^{p} v_{t} d x+\int_{\Omega}|\nabla v|^{2} d x=\alpha \int_{\Omega} v^{r+1} d x
$$

that is

$$
\frac{p}{p+1} \frac{d}{d t}\|v(t)\|_{p+1}^{p+1}+2 J^{*}(v(t))=\alpha \frac{r-1}{r+1} \int_{\Omega} v^{r+1} d x .
$$

As the mapping $t \longmapsto J^{*}(v(t))$ is decreasing, we get $\frac{p}{p+1} \frac{d}{d t}\|v(t)\|_{p+1}^{p+1}+2 J^{*}\left(v_{0}\right) \geq 0$; we deduce immediately $(2.11)$.

Now we show that if $J^{*}\left(v_{0}\right) \leq 0$ the solution of $(2.1)$ tends to $+\infty$ in the case $r \leq p$ or blows up in a finite time in the case $r>p$. 
Theorem 2.4. If $J^{*}\left(v_{0}\right) \leq 0$ and $r \leq p$, then $\lim _{t \rightarrow+\infty}\|v(t)\|_{p+1}=+\infty$, except in the case $r=1$ and $J^{*}\left(v_{0}\right)=0$.

If $J^{*}\left(v_{0}\right) \leq 0$ and $r>p$, the solution of (2.1) blows up in a finite time $T_{b}$ such that

$$
T_{1} \leq T_{b} \leq T_{2}
$$

with

$$
T_{2}=\frac{1}{\alpha} \frac{p}{r-p} \frac{r+1}{r-1} \frac{1}{C_{2}(\Omega)\left\|v_{0}\right\|_{p+1}^{r-p}},
$$

where $C_{2}(\Omega)$ is a positive constant depending only on $\Omega$.

Moreover, for $t \in\left[0, T_{b}[\right.$, we have the inequality

$$
\left\|v_{0}\right\|_{p+1}\left[\frac{T_{2}}{T_{2}-t}\right]^{\frac{1}{r-p}} \leq\|v(t)\|_{p+1} .
$$

Proof. Let us prove the result first in the case $r<p$. The relation (2.12) may also be written as

$$
\frac{p}{p+1} \frac{d}{d t}\|v(t)\|_{p+1}^{p+1}=-(r+1) J^{*}(v(t))+\frac{r-1}{2} \int_{\Omega}|\nabla v(t)|^{2} d x
$$

as the mapping $t \longmapsto J^{*}(v(t))$ is decreasing, and by using the positive constant defined in (2.3), we get

$$
\frac{p}{p+1} \frac{d}{d t}\|v(t)\|_{p+1}^{p+1}+(r+1) J^{*}\left(v_{0}\right) \geq \frac{r-1}{2} C(\Omega)\|v(t)\|_{p+1}^{2} .
$$

If $J^{*}\left(v_{0}\right) \leq 0$, this inequality leads to $\|v(t)\|_{p+1}^{p-1} \geq\left\|v_{0}\right\|_{p+1}^{p-1}+\frac{(p-1)(r-1)}{2 p} C(\Omega) t$, then $\lim _{t \longrightarrow+\infty}\|v(t)\|_{p+1}=+\infty$.

If $r>p$, we use the equality (2.13) and we get

$$
\frac{p}{p+1} \frac{d}{d t}\|v(t)\|_{p+1}^{p+1}+2 J^{*}\left(v_{0}\right) \geq \alpha \frac{r-1}{r+1}\|v(t)\|_{r+1}^{r+1} .
$$

By using the Hölder inequality

$$
\|v\|_{r+1}^{r+1} \geq C_{2}(\Omega)\|v\|_{p+1}^{r+1} \quad \forall v \in L^{r+1}(\Omega) \text { with } C_{2}(\Omega)=(\operatorname{mes} \Omega)^{-\frac{r-p}{p+1}},
$$

we deduce if $J^{*}\left(v_{0}\right) \leq 0$, then

$$
\frac{d}{d t}\|v(t)\|_{p+1}^{-(r-p)} \leq-\alpha \frac{r-1}{r+1} \frac{r-p}{p} C_{2}(\Omega)
$$

and by integrating in time we get

$$
\left\|v_{0}\right\|_{p+1}^{r-p}\left[1-\alpha \frac{r-1}{r+1} \frac{r-p}{p} C_{2}(\Omega)\left\|v_{0}\right\|_{p+1}^{r-p} t\right]^{-1} \leq\|v(t)\|_{p+1}^{r-p} .
$$

The first member of this inequality becomes infinite at the time $T_{2}$. We deduce that $T_{2}$ is a bound on the maximal time of existence, and by using the same argument as Levine-Sacks ([13]), we obtain that the solution blows up at a time $T_{b} \leq T_{2}$ and from Lemma 2.1 such that $T_{b} \geq T_{1}$. 
Theorem 2.5. If $r \leq p$ and $v_{0}$ satisfies

$$
\alpha\left\|v_{0}\right\|_{p+1}^{r-1}<C(\Omega) / C_{2}(\Omega),
$$

then the solution of (2.1) vanishes in a finite time $T_{e}$ such that

$$
T_{e_{1}} \leq T_{e} \leq T_{e_{2}}
$$

with

$$
T_{e_{1}}=\frac{1}{2} \frac{p}{p+1} \frac{\left\|v_{0}\right\|_{p+1}^{p+1}}{J^{*}\left(v_{0}\right)} \quad \text { and } \quad T_{e_{2}}=\frac{p}{p-1} \frac{\left\|v_{0}\right\|_{p+1}^{p-1}}{C(\Omega)-C_{2}(\Omega) \alpha\left\|v_{0}\right\|_{p+1}^{r-1}} .
$$

Proof. From the relation (2.12) we get

$$
\frac{p}{p+1} \frac{d}{d t}\|v(t)\|_{p+1}^{p+1}+C(\Omega)\|v(t)\|_{p+1}^{2} \leq \alpha\|v(t)\|_{r+1}^{r+1} .
$$

The right side can be bounded by using the Hölder inequality

$$
\|v\|_{r+1}^{r+1} \leq C_{2}(\Omega)\|v\|_{p+1}^{r+1} \quad \forall v \in L_{p+1}(\Omega) .
$$

Thus by denoting $\varphi(t)=C(\Omega)-\alpha C_{2}(\Omega)\|v(t)\|_{p+1}^{r-1}$, we get

$$
\frac{d}{d t}\|v(t)\|_{p+1}^{p-1} \leq-\frac{p-1}{p} \varphi(t) .
$$

We can check that if $\varphi(0)>0$, namely $\alpha C_{2}(\Omega)\left\|v_{0}\right\|_{p+1}^{r-1}<C(\Omega)$, the mapping $t \longmapsto$ $\varphi(t)$ is increasing, which in addition to $(2.24)$ implies $\frac{d}{d t}\|v(t)\|_{p+1}^{p-1} \leq-\frac{p-1}{p} \varphi(0)$. By integrating in time we get

$$
\|v(t)\|_{p+1}^{p-1} \leq\left\|v_{0}\right\|_{p+1}^{p-1}-\frac{p-1}{p} \varphi(0) t .
$$

If the right side of this inequality becomes null in a finite time, then the solution $v$ vanishes in a finite time $T_{e}$ such that $T_{e} \leq T_{e_{2}}$.

From Theorem 2.4, the solution cannot vanish if $J^{*}\left(v_{0}\right) \leq 0$; hence the left inequality in (2.21) proceeds easily from (2.11).

Theorem 2.6. If $r>p$ and $v_{0}$ satisfies

$$
\alpha \frac{r-1}{p-1}\left\|v_{0}\right\|_{\infty}^{r-p}\left\|v_{0}\right\|_{p+1}^{p-1} \leq C(\Omega),
$$

the solution of (2.1) vanishes in a finite time $T_{e}$ such that

$$
T_{e_{1}} \leq T_{e} \leq T_{e_{3}}
$$

with

$$
T_{e_{3}}=T_{1}-T_{1}\left[1-\frac{\alpha(r-1)}{C(\Omega)(p-1)}\left\|v_{0}\right\|_{\infty}^{r-p}\left\|v_{o}\right\|_{p+1}^{p-1}\right]^{\frac{r-p}{r-1}} .
$$

Proof. From the relation (2.12) we get

$$
\frac{p}{p+1} \frac{d}{d t}\|v(t)\|_{p+1}^{p+1}+C(\Omega)\|v(t)\|_{p+1}^{2} \leq \alpha\|v(t)\|_{\infty}^{r-p}\|v(t)\|_{p+1}^{p+1} .
$$

Hence we deduce

$$
\frac{p}{p-1} \frac{d}{d t}\|v(t)\|_{p+1}^{p-1}+C(\Omega) \leq \alpha\|v(t)\|_{\infty}^{r-p}\|v(t)\|_{p+1}^{p-1}
$$


We use the estimate (2.7) to obtain

$$
\frac{d}{d t}\|v(t)\|_{p+1}^{p-1}+\frac{p-1}{p} C(\Omega) \leq \alpha \frac{p-1}{p} \frac{T_{1}}{T_{1}-t}\left\|v_{0}\right\|_{\infty}^{r-p}\|v(t)\|_{p+1}^{p-1} .
$$

Let us denote $C_{1}=\frac{p-1}{p} C(\Omega), \mu=\frac{p-1}{r-p}$; this inequality may be written as

$$
\frac{d}{d t}\left(\left(T_{1}-t\right)^{\mu}\|v(t)\|_{p+1}^{p-1}\right) \leq-C_{1}\left(T_{1}-t\right)^{\mu},
$$

and by integrating in time between 0 and $t$, it follows that

$$
\|v(t)\|_{p+1}^{p-1} \leq\left[\left\|v_{0}\right\|_{p+1}^{p-1}-\frac{C_{1} T_{1}}{\mu+1}\right] \frac{T_{1}^{\mu}}{\left(T_{1}-t\right)^{\mu}}+\frac{C_{1}}{\mu+1}\left(T_{1}-t\right) .
$$

If $v_{0}$ satisfies $\left\|v_{0}\right\|_{p+1}^{p-1} \leq \frac{C_{1} T_{1}}{\mu+1}$, namely if $\alpha \frac{r-1}{p-1}\left\|v_{0}\right\|_{\infty}^{r-p}\left\|v_{0}\right\|_{p+1}^{p-1} \leq C(\Omega)$, the right side of the inequality (2.28) becomes null at the time $T_{e_{2}}$ such that

$$
\left(T_{1}-T_{e_{2}}\right)^{\mu+1}=T_{1}^{\mu+1}\left[1-(\mu+1) \frac{\left\|v_{0}\right\|_{p+1}^{p-1}}{C_{1} T_{1}}\right] .
$$

We conclude that the function $v$ becomes null in a finite time $T_{e} \leq T_{e_{2}}$.

The left inequality of (2.26) again proceeds from (2.11).

In the particular case $r=1$ (i.e., $p_{1}=m$ ) we have more accurate results.

We introduce the functional $F$ defined by

$$
F(\varphi)=\frac{1}{\|\varphi\|_{p+1}^{2}} \int_{\Omega}\left(|\nabla \varphi|^{2}-\alpha \varphi^{2}\right) d x=\frac{2 J^{*}(\varphi)}{\|\varphi\|_{p+1}^{2}} \quad \forall \varphi \in H_{0}^{1}(\Omega) .
$$

Let us denote $\lambda_{1}$ the first eigenvalue of the Dirichlet problem $-\Delta \rho=\lambda \rho, x \in \Omega$, $\rho=0, x \in \partial \Omega$.

We have the inequality $F(\varphi) \geq C(\Omega) \frac{\lambda_{1}-\alpha}{\lambda_{1}}$, and since

$$
\frac{d}{d t}\|v(t)\|_{p+1}^{p-1}=-\frac{p-1}{p} F(v(t))
$$

we get

$$
\|v(t)\|_{p+1}^{p-1} \leq\left\|v_{0}\right\|_{p+1}^{p-1}-\frac{p-1}{p} \frac{\lambda_{1}-\alpha}{\lambda_{1}} C(\Omega) t .
$$

Then, if $\alpha<\lambda_{1}$, the second member becomes null in a finite time, so the solution vanishes in a finite time $T_{e}$ such that

$$
T_{e} \leq \frac{p}{p-1} \frac{\lambda_{1}}{\lambda_{1}-\alpha} \frac{\left\|v_{0}\right\|_{p+1}^{p-1}}{C(\Omega)}
$$

We easily prove that the mapping $t \longmapsto F(v(t))$ is decreasing, then from (2.30), we get

$$
\|v(t)\|_{p+1}^{p-1} \geq\left\|v_{0}\right\|_{p+1}^{p-1}-\frac{p-1}{p} F\left(v_{0}\right) t .
$$

In the case $\lambda_{1}>0$, if $F\left(v_{0}\right)<0$, then we obtain immediately that $\lim _{t \longrightarrow+\infty}\|v(t)\|_{p+1}=$ $+\infty$; if it is not the case, by using the same argument as Friedman and McLeod in [6], we prove again that $\lim _{t \longrightarrow+\infty}\|v(t)\|_{p+1}=+\infty([14])$. 
If $\alpha=\lambda_{1}$, then the problem has a global solution which tends to $\theta \rho_{1}$ as $t \longrightarrow+\infty$ ( $\rho_{1}$ is the eigenfunction corresponding to $\lambda_{1}$ satisfying $\rho_{1}>0,\left\|\rho_{1}\right\|_{L^{1}(\Omega)}=1$ and $\left.\theta=\left(\int_{\Omega} v_{0}^{p} \rho_{1} d x\right)^{\frac{1}{p}}\right)$. The proof uses the same argument as Sacks in [18].

In the particular case $r=p$ (i.e., $p_{1}=1$ ) we also have more precise results (see [10]).

We define the functional $G$ as

$$
G(\varphi)=\frac{1}{\|\varphi\|_{p+1}^{2}} \int_{\Omega}|\nabla \varphi|^{2} d x, \forall \varphi \in H_{0}^{1}(\Omega)
$$

and we prove that the mapping $t \longmapsto G(v(t))$ is decreasing. From the equality

$$
\frac{p}{p-1} \frac{d}{d t}\|v(t)\|_{p+1}^{p-1}+G(v(t))=\alpha\|v(t)\|_{p+1}^{p-1}
$$

we get

$$
\exp \left(-\alpha \frac{p-1}{p} t\right)\|v(t)\|_{p+1}^{p-1}+\frac{p-1}{p} \int_{0}^{t} G(v(s)) \exp \left(-\alpha \frac{p-1}{p} s\right) d s=\left\|v_{0}\right\|_{p+1}^{p-1},
$$

and since the function $t \longmapsto G(v(t))$ is decreasing, we obtain

$$
\|v(t)\|_{p+1}^{p-1} \geq \exp \left(\alpha \frac{p-1}{p} t\right)\left(\left\|v_{0}\right\|_{p+1}^{p-1}-\frac{G\left(v_{0}\right)}{\alpha}\right)+\frac{G\left(v_{0}\right)}{\alpha}
$$

$$
\|v(t)\|_{p+1}^{p-1} \leq \exp \left(\alpha \frac{p-1}{p} t\right)\left(\left\|v_{0}\right\|_{p+1}^{p-1}-\frac{G(v(t))}{\alpha}\right)+\frac{G(v(t))}{\alpha} .
$$

Since $G(v(t)) \geq C(\Omega)$, if $\alpha\left\|v_{0}\right\|_{p+1}^{p-1}<C(\Omega)$, the solution vanishes in a finite time $T_{e}$ such that

$$
T_{e} \leq \frac{p}{\alpha(p-1)} \ln \left(\frac{C(\Omega)}{C(\Omega)-\alpha\left\|v_{0}\right\|_{p+1}^{p-1}}\right) .
$$

If $\alpha\left\|v_{0}\right\|_{p+1}^{p-1}>G\left(v_{0}\right)$, then the solution tends to infinity as $t \longrightarrow+\infty$.

\section{Definition OF the NUMERICAL SCHEME}

If we use a classical Euler scheme for the semidiscretization in time of the problem, the corresponding numerical solution cannot vanish or blow up after a finite number of time steps.

So, we generalize here the numerical scheme used in [8]: if $v_{n}$ is the approximate value of the solution at the time level $t_{n}=n \Delta t$ ( $\Delta t$ is the time step), then $v_{n+1}$ is the solution of the equation

$$
\frac{p}{p-1} v_{n+1}\left(v_{n+1}^{p-1}-v_{n}^{p-1}\right)+\Delta t A v_{n+1}=\Delta t\left(\alpha_{1} v_{n+1} v_{n}^{r-1}+\alpha_{2} v_{n+1}^{p} v_{n}^{r-p}\right)
$$

with $\alpha_{1}+\alpha_{2}=\alpha, \alpha_{1}, \alpha_{2} \geq 0$.

If $r \leq p$, we choose $\alpha_{2}=0, \alpha_{1}=\alpha$ in order to avoid negative powers in the second member.

If $r>p$, if $\alpha_{2}=0$, the solution of (3.1) may become null in a finite time, but it cannot blow up in a finite time when this physical peculiarity appears for the continuous problem. If $\alpha_{1}=0$, the solution may become null or infinite in a finite time, but this solution is not always bounded by the solution of the differential 
equation (2.8) as it is the case for the exact solution; it depends on the values of $r$ and $p$. So we choose $\alpha_{1}$ and $\alpha_{2}$ such that the numerical solution is bounded by the solution of the differential equation and is as close as possible to this solution.

This leads us to consider the following values of $\alpha_{1}$ and $\alpha_{2}$ :

$$
\begin{gathered}
\alpha_{1}=\alpha, \alpha_{2}=0 \quad \text { if } r \leq p, \\
\alpha_{1}=\frac{2 p-1-r}{p-1} \alpha, \quad \alpha_{2}=\frac{r-p}{p-1} \alpha \quad \text { if } p \leq r \leq 2 p-1, \\
\alpha_{1}=0, \alpha_{2}=\alpha \quad \text { if } r \geq 2 p-1 .
\end{gathered}
$$

Lemma 3.1. If the solution $v_{n}$ is positive on $\Omega, v_{n} \in H_{0}^{1}(\Omega) \cap C^{\epsilon}(\bar{\Omega}),(\epsilon \in] 0,1[)$ and satisfies

$$
\alpha_{2} \frac{p-1}{p} \Delta t\left\|v_{n}\right\|_{\infty}^{r-p}<1 \text { in the case } r>p,
$$

then the problem (3.1) has a maximal nonnegative solution $\bar{v} \in H_{0}^{1}(\Omega) \cap C^{2}(\bar{\Omega})$. Besides every nonnegative solution $v$ satisfies $0 \leq v \leq \bar{v}$.

Proof. The problem (3.1) may be written as $A v_{n+1}=f\left(v_{n+1}\right)$, where $f$ is the function defined by

$$
f(u)=\frac{p}{(p-1) \Delta t}\left[\left(1+\alpha_{1} \frac{p-1}{p} \Delta t v_{n}^{r-p}\right) v_{n}^{p-1} u-\left(1-\alpha_{2} \frac{p-1}{p} \Delta t v_{n}^{r-p}\right) u^{p}\right],
$$

where $f$ satisfies $f(0)=0$.

In addition, if (3.3) is satisfied, (3.1) has a constant supersolution $C_{n}$ such that

$$
C_{n}^{p-1}=\frac{\left\|v_{n}\right\|_{\infty}^{p-1}+\alpha_{1} \frac{p-1}{p} \Delta t\left\|v_{n}\right\|_{\infty}^{r-1}}{1-\alpha_{2} \frac{p-1}{p} \Delta t\left\|v_{n}\right\|_{\infty}^{r-p}} .
$$

Hence we deduce from a result from Amann [1] that (3.1) has a minimal solution (the null solution) and a maximal solution $\bar{v} \in C_{2}(\bar{\Omega})$. Besides any solution satisfies $v \in C^{2}(\bar{\Omega})$ and $0 \leq v \leq \bar{v}$.

Lemma 3.2. Under the hypothesis of the previous lemma, (3.1) has at most one positive solution.

The proof is the same as in [8].

In order to set up a sufficient condition for the solution of the numerical sheme to be positive, we introduce the functional $F$, which is defined by

$$
F(\varphi)=\frac{1}{\|\varphi\|_{p+1}^{2}} \int_{\Omega}\left(|\nabla \varphi|^{2}-\alpha_{1} \varphi^{r+1}\right) d x, \quad \forall \varphi \in H_{0}^{1}(\Omega) \cap L^{r+1}(\Omega) .
$$

Lemma 3.3. Under the hypothesis of the Lemma 3.1 and if $v_{n}$ satisfies

$$
\frac{p-1}{p} \Delta t F\left(v_{n}\right)<\left\|v_{n}\right\|_{p+1}^{p-1},
$$

then (3.1) has a positive solution in $H_{0}^{1}(\Omega) \cap C^{2}(\bar{\Omega})$. 
Proof. Let us introduce the set $K$ and the functional $J_{n}$ on $H_{0}^{1}(\Omega)$

$$
\begin{gathered}
K=\left\{v \in H_{0}^{1}(\Omega) / \int_{\Omega}\left(1-\alpha_{2} \frac{p-1}{p} \Delta t v_{n}^{r-p}\right)|v|^{p+1} d x=1\right\}, \\
J_{n}(v)=\int_{\Omega}|\nabla v|^{2} d x-\frac{p}{(p-1) \Delta t} \int_{\Omega} v_{n}^{p-1}\left(1+\alpha_{1} \frac{p-1}{p} \Delta t v_{n}^{r-p}\right) v^{2} d x,
\end{gathered}
$$

and let us consider the minimization problem $\varphi_{n} \in K, J_{n}\left(\varphi_{n}\right)=\min _{v \in K} J_{n}(v)$. By using a Hölder inequality, we easily set

$$
J_{n}(v) \geq-\frac{p}{(p-1) \Delta t} \int_{\Omega} v_{n}^{p+1} \frac{\left(1+\alpha_{1} \frac{p-1}{p} \Delta t v_{n}^{r-p}\right)^{\frac{p+1}{p-1}}}{\left(1-\alpha_{2} \frac{p-1}{p} \Delta t v_{n}^{r-p}\right)^{\frac{2}{p-1}}} d x, \forall v \in K .
$$

Hence, $J_{n}$ has a lower bound on $K$.

Let $\varphi_{n, k}$ be a minimizing sequence; since $J_{n}\left(\left|\varphi_{n, k}\right|\right)=J\left(\varphi_{n, k}\right)$, we may suppose $\varphi_{n, k>0}$. This sequence is bounded in $H_{0}^{1}(\Omega)$, so we can extract a subsequence, again labeled $\varphi_{n, k}$, which converges to $\varphi_{n}$ weakly in $H_{0}^{1}(\Omega)$ and strongly in $L^{p+1}(\Omega)$. Hence we have $\varphi_{n} \geq 0, \varphi_{n} \in K$ and for all $v \in H_{0}^{1}(\Omega)$, we obtain

$$
A \varphi_{n}-\left(\frac{p}{(p-1) \Delta t} v_{n}^{p-1}+\alpha_{1} v_{n}^{r-1}\right) \varphi_{n}=J_{n}\left(\varphi_{n}\right)\left(1-\alpha_{2} \frac{p-1}{p} \Delta t v_{n}^{r-p}\right) \varphi_{n}^{p} .
$$

Then, if $J_{n}\left(\varphi_{n}\right)<0$, the solution $v_{n+1}$ of the problem (3.1) is defined by

$$
v_{n+1}=\left(-\frac{p-1}{p} \Delta t J_{n}\left(\varphi_{n}\right)\right)^{\frac{1}{p-1}} \varphi_{n} .
$$

It remains to determine a sufficient condition for $J_{n}\left(\varphi_{n}\right)<0$.

Since the function $\psi_{n}=\left(\int_{\Omega}\left(v_{n}^{p+1}-\alpha_{2} \frac{p-1}{p} \Delta t v_{n}^{r+1}\right) d x\right)^{-\frac{1}{p+1}} v_{n}$ belongs to $K$, we necessarily have $J_{n}\left(\varphi_{n}\right) \leq J_{n}\left(\psi_{n}\right)$, where

$$
\begin{aligned}
J_{n}\left(\psi_{n}\right)=\left\|v_{n}\right\|_{p+1}^{2} & \left(F\left(v_{n}\right)-\frac{p}{(p-1) \Delta t}\left\|v_{n}\right\|_{p+1}^{p-1}\right) \\
\times & \left(\int_{\Omega}\left(v_{n}^{p+1}-\alpha_{2} \frac{p-1}{p} \Delta t v_{n}^{r+1}\right) d x\right)^{-\frac{2}{p+1}} .
\end{aligned}
$$

Hence if $J_{n}\left(\psi_{n}\right)<0$, namely if $\frac{p-1}{p} \Delta t F\left(v_{n}\right)<\left\|v_{n}\right\|_{p+1}^{p-1}$, then we obtain $J_{n}\left(\varphi_{n}\right)<0$ and (3.1) admits one positive solution.

Now we prove that the numerical solution is bounded by one of the differential equations (2.8), as it is true for the exact solution.

Lemma 3.4. If the parameters $\alpha_{1}$ and $\alpha_{2}$ are chosen as in (3.2) and if the hypotheses of Lemmas 3.1 and 3.3 are satisfied, we have the inequality

$$
\left\|v_{n}\right\|_{\infty} \leq\left\|v_{0}\right\|_{\infty}\left(1+\alpha \frac{p-r}{p}\left\|v_{0}\right\|_{\infty}^{r-p} t_{n}\right)^{\frac{1}{p-r}} \quad \text { if } r \neq p,
$$




$$
\left\|v_{n}\right\|_{\infty} \leq\left\|v_{0}\right\|_{\infty} \exp \left(\frac{\alpha}{p} t_{n}\right)
$$

Proof. We denote by $w_{n}$ the solution of the differential equation (2.8) at the time level $t_{n}=n \Delta t$,

$$
\begin{gathered}
w_{n}=w_{0}\left(1+\alpha \frac{p-r}{p} \Delta t w_{0}^{r-p}\right)^{\frac{1}{p-r}} \text { if } r \neq p, \\
w_{n}=w_{0} \exp \left(\frac{\alpha}{p} t_{n}\right),
\end{gathered}
$$

and we prove the lemma recurrently: the result is true for $n=0$ since $w(0)=\left\|v_{0}\right\|_{\infty}$. Then $w_{n+1}$ is a supersolution of $(3.1)$ if $f\left(w_{n+1}\right) \leq 0$; namely

$$
v_{n}^{p-1}\left(1+\alpha_{1} \frac{p-1}{p} \Delta t v_{n}^{r-p}\right) \leq w_{n+1}^{p-1}\left(1-\alpha_{2} \frac{p-1}{p} \Delta t v_{n}^{r-p}\right),
$$

that is

$$
\begin{aligned}
& v_{n}^{p-1}\left(1+\alpha_{1} \frac{p-1}{p} \Delta t v_{n}^{r-p}\right) \\
& \quad \leq w_{n}^{p-1}\left(1+\alpha \frac{p-r}{p} \Delta t w_{n}^{r-p}\right)^{\frac{p-1}{p-r}}\left(1-\alpha_{2} \frac{p-1}{p} \Delta t v_{n}^{r-p}\right) \quad \text { if } r \neq p
\end{aligned}
$$

and

$$
v_{n}^{p-1}\left(1+\alpha \frac{p-1}{p} \Delta t\right) \leq w_{n}^{p-1} \exp \left(\frac{\alpha(p-1)}{p} \Delta t\right) \quad \text { if } r=p .
$$

As $\left\|v_{n}\right\|_{\infty} \leq w_{n}$, in the case $r=p$, the above inequality is immediately verified and in the case $r \neq p$ it will be true if

$$
1+\alpha_{1} \frac{p-1}{p} \Delta t w_{n}^{r-p} \leq\left(1-\alpha_{2} \frac{p-1}{p} \Delta t w_{n}^{r-p}\right)\left(1+\alpha \frac{p-r}{p} \Delta t w_{n}^{r-p}\right)^{\frac{p-1}{p-r}} .
$$

Let us prove that this last inequality holds if $\alpha_{1}$ and $\alpha_{2}$ are chosen as in (3.2). For this, we consider the mapping $h: x \longmapsto\left(1-\alpha_{2} x\right)\left(1+\alpha \frac{x}{\mu}\right)$, where $\mu=\frac{p-1}{p-r}, r \neq p$. This function is defined for $x \geq 0$ if $r<p$ and for $-\alpha \frac{x}{\mu} \leq 1$ if $r>p$. Moreover, it is easy to check that $h^{\prime \prime}(x) \geq 0 \forall x \in\left[0, \frac{-\mu}{\alpha}\right.$. So we have the inequality $h(0)+x h^{\prime}(0) \leq$ $h(x)$. In particular, for $x=\frac{p-1}{p} \Delta t w_{n}^{r-p}$, we obtain the inequality (3.15). We deduce $\left\|v_{n+1}\right\|_{\infty} \leq w_{n+1}$, which achieves the proof.

Computation of the positive solution of the numerical scheme. Equation (3.1) may be written as

$$
A v_{n+1}=f\left(v_{n+1}\right)
$$

where $f$ is the function defined in (3.4).

In order to compute a numerical solution of (3.1), we use a result of Keller [7]. The function $f$ satisfies the inequality $f(v)-f(u) \geq-m(v-u)$, where $m$ is the function defined on $\Omega$ by

$$
m=\frac{p}{(p-1) \Delta t}\left(p C_{n}^{p-1}\left(1-\alpha_{2} \frac{p-1}{p} \Delta t v_{n}^{r-p}\right)-v_{n}^{p-1}\left(1+\alpha_{1} \frac{p-1}{p} \Delta t v_{n}^{r-p}\right)\right)
$$

with $C_{n}$ defined in (3.5). 
So if we define the sequence $\left(v_{n+1, j}\right)_{j \geq 0}$ by

$$
(A+m I) v_{n+1, j+1}=f\left(v_{n+1, j}\right)+m v_{n+1, j}
$$

or

$$
(A+m I) v_{n+1, j+1}=\frac{p}{(p-1) \Delta t}\left(1-\alpha_{2} \frac{p-1}{p} \Delta t v_{n}^{r-p}\right)\left(p C_{n}^{p-1}-v_{n+1, j}^{p-1}\right) v_{n+1, j},
$$

we obtain a monotone sequence if the first iterate is a subsolution or a supersolution; it is the choice of $v_{n+1,0}$ that determines whether the sequence is decreasing or increasing. This sequence converges to the solution. For example, we can choose $v_{n+1,0}=C_{n}$ since $C_{n}$ is a supersolution and the sequence $\left(v_{n+1, j}\right)_{j \geq 0}$ is a decreasing sequence converging to $v_{n+1}$.

If $r>p$, the constant $C_{n}$ may be very large when $t_{n}$ is close to $T_{1}$. So we shall prove that in this case we again obtain a convergent sequence $\left(v_{n+1, j}\right)_{j \geq 0}$ by choosing $v_{n+1,0}=v_{n}$.

In order to prove this result, we need the following lemma.

Lemma 3.5. For $n \geq 1$, we have the inequality

$$
v_{n+1} \leq\left(\frac{2+\alpha_{1} \frac{p-1}{p} \Delta t\left\|v_{n}\right\|_{\infty}^{r-p}}{1-\alpha_{2} \frac{p-1}{p} \Delta t\left\|v_{n}\right\|_{\infty}^{r-p}}\right)^{\frac{1}{p-1}} v_{n} \quad \text { if } r \geq p .
$$

Proof. Let $\tau \geq 1$; the function $\tau^{\frac{1}{p-1}} v_{n}$ will be a supersolution of (3.1) if

$$
A v_{n} \geq \frac{p}{(p-1) \Delta t}\left(\left(v_{n}^{p}+\alpha_{1} \frac{p-1}{p} \Delta t v_{n}^{r}\right)-\tau\left(v_{n}^{p}-\alpha_{2} \frac{p-1}{p} \Delta t v_{n}^{r}\right)\right),
$$

but

$$
A v_{n}=\frac{p}{(p-1) \Delta t}\left(v_{n-1}^{p-1}\left(1+\alpha_{1} \frac{p-1}{p} \Delta t v_{n-1}^{r-p}\right) v_{n}-\left(1-\alpha_{2} \frac{p-1}{p} \Delta t v_{n-1}^{r-p}\right) v_{n}^{p}\right),
$$

which implies $A v_{n} \geq-\frac{p}{(p-1) \Delta t} v_{n}^{p}$. Then for $n \geq 1$, the inequality will hold if

$$
\frac{p}{(p-1) \Delta t}(\tau-2) v_{n}^{p} \geq \alpha_{1} v_{n}^{r}+\tau \alpha_{2} v_{n}^{r},
$$

which will be true if

$$
\tau \geq \frac{2+\alpha_{1} \frac{p-1}{p} \Delta t\left\|v_{n}\right\|_{\infty}^{r-p}}{1-\alpha_{2} \frac{p-1}{p} \Delta t\left\|v_{n}\right\|_{\infty}^{r-p}}
$$

Theorem 3.6. If $r \geq p$ and $v_{n+1,0}=v_{n}(n \geq 1)$, the sequence $\left(v_{n+1, j}\right)_{j \geq 0}$ converges uniformly to $v_{n+1}$.

Proof. Let us consider the sequences $\left(w_{j}\right)_{j \geq 0},\left(z_{j}\right)_{j \geq 0}$ obtained from (3.18) with the following respective initial values:

$$
w_{0}=\left(\frac{1-\alpha_{2} \frac{p-1}{p} \Delta t\left\|v_{n}\right\|_{\infty}^{r-p}}{2+\alpha_{1} \frac{p-1}{p} \Delta t\left\|v_{n}\right\|_{\infty}^{r-p}}\right)^{\frac{1}{p-1}} v_{n+1,} \quad z_{0}=C_{n} .
$$

The following inequalities hold: $w_{0} \leq v_{n} \leq z_{0}$ (see (3.19)). Since the operator $(A+m I)$ is monotone and the mapping $u \longmapsto f(u)+m u$ is increasing, we deduce that $w_{j} \leq v_{n+1, j} \leq z_{j}$. 
Besides, since $w_{0}$ is a subsolution of (3.1), the sequence $\left(w_{j}\right)_{j \geq 0}$ is a monotone increasing sequence converging uniformly to $v_{n+1}$. In the same way, since $z_{0}$ is a supersolution of (3.1), the sequence $\left(z_{j}\right)_{j \geq 0}$ is a monotone decreasing sequence converging uniformly to $v_{n+1}$. Therefore, the sequence $\left(w_{j}\right)_{j \geq 0}$ converges uniformly to $v_{n+1}$.

Remark 3.7. We also observe that the sequence $\left(v_{n+1, j}\right)_{j \geq 0}$ converges more rapidly by taking $v_{n+1,0}=v_{n}$ in the case $r<p$.

\section{Properties of the nUmerical SOlution}

In this part, we check that the numerical solution has the same properties as the exact solution. We prove that the solution of the numerical scheme exists and remains positive during at least a finite lapse of time and we obtain sufficient conditions for this solution to become infinite or null in a finite time.

Lemma 4.1. For $n \geq 0$, we have the inequality

$$
\begin{aligned}
& \frac{p-1}{p} \Delta t \frac{1}{\left\|v_{n+1}\right\|_{p+1}^{2}} \int_{\Omega}\left(\left|\nabla v_{n+1}\right|^{2}-\alpha_{2} v_{n}^{r-p} v_{n+1}^{p+1}-\alpha_{1} v_{n}^{r-1} v_{n+1}^{2}\right) d x \\
& \quad \leq\left\|v_{n}\right\|_{p+1}^{p-1}-\left\|v_{n+1}\right\|_{p+1}^{p-1} .
\end{aligned}
$$

Proof. By multiplying equation (3.1) by $v_{n+1}$ and integrating on $\Omega$, we get

$$
\begin{array}{r}
\frac{p}{p-1} \int_{\Omega} v_{n+1}^{2}\left(v_{n+1}^{p-1}-v_{n}^{p-1}\right) d x+\Delta t \int_{\Omega}\left|\nabla v_{n+1}\right|^{2} d x \\
=\Delta t\left(\alpha_{1} \int_{\Omega} v_{n}^{r-1} v_{n+1}^{2} d x+\alpha_{2} \int_{\Omega} v_{n}^{r-p} v_{n+1}^{p+1} d x\right) .
\end{array}
$$

Besides, we have the inequality

$$
\int_{\Omega} v_{n+1}^{2}\left(v_{n+1}^{p-1}-v_{n}^{p-1}\right) d x \geq\left\|v_{n+1}\right\|_{p+1}^{2}\left(\left\|v_{n+1}\right\|_{p+1}^{p-1}-\left\|v_{n}\right\|_{p+1}^{p-1}\right) .
$$

We deduce the result.

Lemma 4.2. For $n \geq 0$, we have the inequalities.

$$
\text { If } r \leq p,
$$

$$
\begin{aligned}
& \left\|v_{n}\right\|_{p+1}^{p-1}-\left\|v_{n+1}\right\|_{p+1}^{p-1} \leq \frac{p-1}{p} \Delta t \frac{1}{\left\|v_{n}\right\|_{p+1}^{2}} \int_{\Omega}\left(\left|\nabla v_{n}\right|^{2}-\alpha v_{n}^{r+1}\right) d x . \\
& \text { If } r>p \text {, } \\
& \left\|v_{n}\right\|_{p+1}^{p+1}-\left\|v_{n+1}\right\|_{p+1}^{p+1} \\
& \leq \frac{p+1}{p} \Delta t\left(\int_{\Omega}\left(\left|\nabla v_{n}\right|^{2}-\alpha v_{n}^{r+1}\right) d x+\alpha_{2} \frac{p-1}{p+1} \int_{\Omega} v_{n}^{r-p}\left(v_{n}^{p+1}-v_{n+1}^{p+1}\right) d x\right) .
\end{aligned}
$$


Proof. From Lemma 3.3, the solution of (3.1) is written as

$$
v_{n+1}=\left(-\frac{p-1}{p} \Delta t J_{n}\left(\varphi_{n}\right)\right)^{\frac{1}{p-1}} \varphi_{n}
$$

Moreover, the function $\varphi_{n}$ as an element of K satisfies

$$
\int_{\Omega}\left(1-\alpha_{2} \frac{p-1}{p} \Delta t v_{n}^{r-p}\right) \varphi_{n}^{p+1} d x=1 .
$$

With both the previous arguments, we obtain

$$
\left(\int_{\Omega}\left(1-\alpha_{2} \frac{p-1}{p} \Delta t v_{n}^{r-p}\right) v_{n+1}^{p+1} d x\right)^{\frac{p-1}{p+1}}=-\frac{p-1}{p} \Delta t J_{n}\left(\varphi_{n}\right) .
$$

Besides, from (3.12) we get

$$
J_{n}\left(\varphi_{n}\right) \leq J_{n}\left(v_{n}\right)\left(\int_{\Omega}\left(1-\alpha_{2} \frac{p-1}{p} \Delta t v_{n}^{r-p}\right) v_{n}^{p+1} d x\right)^{-\frac{2}{p+1}}
$$

and we obtain

$$
\begin{aligned}
-\frac{p-1}{p} \Delta t J_{n}\left(v_{n}\right) \leq & \left(\int_{\Omega}\left(1-\alpha_{2} \frac{p-1}{p} \Delta t v_{n}^{r-p}\right) v_{n}^{p+1} d x\right)^{\frac{2}{p+1}} \\
& \times\left(\int_{\Omega}\left(1-\alpha_{2} \frac{p-1}{p} \Delta t v_{n}^{r-p}\right) v_{n+1}^{p+1} d x\right)^{\frac{p-1}{p+1}} .
\end{aligned}
$$

If $r \leq p$, then $\alpha_{2}=0$ and we get

$$
-\frac{p-1}{p} \Delta t J_{n}\left(v_{n}\right) \leq\left\|v_{n}\right\|_{p+1}^{2}\left\|v_{n+1}\right\|_{p+1}^{p-1}
$$

since

$$
J_{n}\left(v_{n}\right)=\int_{\Omega}\left(\left|\nabla v_{n}\right|^{2}-\alpha v_{n}^{r+1}\right) d x-\frac{p}{(p-1) \Delta t}\left\|v_{n}\right\|_{p+1}^{p+1},
$$

we deduce (4.2).

If $r>p$, then $\alpha_{2}>0$ and by using the Young inequality, we get

$$
\begin{aligned}
-\frac{p-1}{p} \Delta t J_{n}\left(v_{n}\right) \leq & \frac{2}{p+1} \int_{\Omega}\left(1-\alpha_{2} \frac{p-1}{p} \Delta t v_{n}^{r-p}\right) v_{n}^{p+1} d x \\
& +\frac{p-1}{p+1} \int_{\Omega}\left(1-\alpha_{2} \frac{p-1}{p} \Delta t v_{n}^{r-p}\right) v_{n+1}^{p+1} d x,
\end{aligned}
$$

and by using the definition of $J_{n}$, we obtain

$$
\begin{gathered}
-\frac{p-1}{p} \Delta t \int_{\Omega}\left(\left|\nabla v_{n}\right|^{2}-\alpha_{1} v_{n}^{r+1}\right) d x \leq \frac{p-1}{p+1}\left(-\left\|v_{n}\right\|_{p+1}^{p+1}+\left\|v_{n+1}\right\|_{p+1}^{p+1}\right) \\
-\frac{p-1}{p} \Delta t \alpha_{2} \int_{\Omega}\left(\frac{2}{p+1} v_{n}^{p+1}+\frac{p-1}{p+1} v_{n+1}^{p+1}\right) v_{n}^{r-p} d x,
\end{gathered}
$$

which gives the result.

Lemma 4.3. For $n \geq 0$, we have

$$
\int_{\Omega} v_{n}^{p-1}\left(v_{n+1}-v_{n}\right)^{2} d x \leq 2 \frac{p+1}{p} \Delta t\left(J^{*}\left(v_{n}\right)-J^{*}\left(v_{n+1}\right)\right) .
$$


Proof. From the following inequality shown in [8],

$$
a^{p-1}(b-a)^{2} \leq a^{p+1}-b^{p+1}+\frac{p+1}{p-1} b^{2}\left(b^{p-1}-a^{p-1}\right),
$$

we deduce that

$$
\int_{\Omega} v_{n}^{p-1}\left(v_{n+1}-v_{n}\right)^{2} d x \leq\left\|v_{n}\right\|_{p+1}^{p+1}-\left\|v_{n+1}\right\|_{p+1}^{p+1}+\frac{p+1}{p-1} \int_{\Omega} v_{n+1}^{2}\left(v_{n+1}^{p-1}-v_{n}^{p-1}\right) d x .
$$

Besides, we have

$$
\begin{aligned}
& \int_{\Omega} v_{n+1}^{2}\left(v_{n+1}^{p-1}-v_{n}^{p-1}\right) d x \\
& \quad=\frac{p-1}{p} \Delta t\left(-\int_{\Omega}\left(\left|\nabla v_{n+1}\right|^{2}-\alpha_{1} v_{n+1}^{2} v_{n}^{r-1}-\alpha_{2} v_{n+1}^{p+1} v_{n}^{r-p}\right) d x\right) .
\end{aligned}
$$

Therefore, the previous inequality becomes

$$
\begin{aligned}
\int_{\Omega} v_{n}^{p-1}\left(v_{n+1}-v_{n}\right)^{2} d x \leq\left\|v_{n}\right\|_{p+1}^{p+1}-\left\|v_{n+1}\right\|_{p+1}^{p+1} \\
\quad+\frac{p+1}{p} \Delta t\left(-\int_{\Omega}\left(\left|\nabla v_{n+1}\right|^{2}-\alpha_{1} v_{n+1}^{2} v_{n}^{r-1}-\alpha_{2} v_{n+1}^{p+1} v_{n}^{r-p}\right) d x\right) .
\end{aligned}
$$

If $r \leq p$, we have the inequality

$$
\left\|v_{n}\right\|_{p+1}^{p+1}-\left\|v_{n+1}\right\|_{p+1}^{p+1} \leq \frac{p+1}{p-1}\left\|v_{n}\right\|_{p+1}^{2}\left(\left\|v_{n}\right\|_{p+1}^{p-1}-\left\|v_{n+1}\right\|_{p+1}^{p-1}\right)
$$

and from (4.2), we get

$$
\left\|v_{n}\right\|_{p+1}^{p+1}-\left\|v_{n+1}\right\|_{p+1}^{p+1} \leq \frac{p+1}{p} \Delta t \int_{\Omega}\left(\left|\nabla v_{n}\right|^{2}-\alpha v_{n}^{r+1}\right) d x .
$$

If $r>p$, we use the inequality (4.3) directly and we obtain $r>p$ and $r \leq p$

$$
\begin{aligned}
\int_{\Omega} v_{n}^{p-1}\left(v_{n+1-} v_{n}\right)^{2} d x \leq & \frac{p+1}{p} \Delta t \int_{\Omega}\left(\left|\nabla v_{n}\right|^{2}-\alpha v_{n}^{r+1}\right) d x \\
& +\alpha_{2} \frac{p-1}{p} \Delta t \int_{\Omega} v_{n}^{r-p}\left(v_{n}^{p+1}-v_{n+1}^{p+1}\right) d x \\
& -\frac{p+1}{p} \Delta t \int_{\Omega}\left(\left|\nabla v_{n+1}\right|^{2}-\alpha_{1} v_{n+1}^{2} v_{n}^{r-1}-\alpha_{2} v_{n+1}^{p+1} v_{n}^{r-p}\right) d x .
\end{aligned}
$$

By using the Young inequality, we easily prove that

$$
\int_{\Omega} v_{n}^{p-1}\left(v_{n+1}-v_{n}\right)^{2} d x \leq 2 \frac{p+1}{p} \Delta t\left(J^{*}\left(v_{n}\right)-J^{*}\left(v_{n+1}\right)\right),
$$

and we deduce that the sequence $\left(J^{*}\left(v_{n}\right)\right)_{n \geq 0}$ is nonincreasing.

Now we state an existence theorem concerning the positive solution of the numerical scheme.

Theorem 4.4. In the case $r \leq p$, if $J^{*}\left(v_{0}\right) \leq 0$, then (3.1) has for $n \geq 0$ a positive solution $v_{n+1} \in H_{0}^{1}(\Omega) \cap C^{2}(\bar{\Omega})$. If $J^{*}\left(v_{0}\right)>0$, then this problem has a positive solution at least until the time $T_{e_{1}}$ defined in (2.22); this solution can become null after this time. 
Proof. For $n \geq 0,(3.1)$ has a maximal solution. We prove that this solution is positive and then unique during a nonempty lapse of time. From (4.2), we have

$$
\left\|v_{n}\right\|_{p+1}^{p+1}-\left\|v_{n+1}\right\|_{p+1}^{p+1} \leq \frac{p+1}{p} \Delta t\left\|v_{n}\right\|_{p+1}^{2} F\left(v_{n}\right)
$$

and $F\left(v_{n}\right)\left\|v_{n}\right\|_{p+1}^{2} \leq 2 J^{*}\left(v_{n}\right)-\alpha \frac{r-1}{r+1}\left\|v_{n}\right\|_{r+1}^{r+1}$.

Since the sequence $\left(J^{*}\left(v_{n}\right)\right)_{n \geq 0}$ is decreasing, we get $F\left(v_{n}\right)\left\|v_{n}\right\|_{p+1}^{2} \leq 2 J^{*}\left(v_{0}\right)$ and we deduce

$$
\left\|v_{0}\right\|_{p+1}^{p+1}-2 \frac{p+1}{p} J^{*}\left(v_{0}\right) t_{n+1} \leq\left\|v_{n+1}\right\|_{p+1}^{p+1} .
$$

So, if $J^{*}\left(v_{0}\right) \leq 0$, the solution $\left(v_{n}\right)_{n \geq 0}$ is positive for any $n \geq 0$, and if $J^{*}\left(v_{0}\right)$ is positive, it is positive at least until the time $T_{e_{1}}$.

We deduce from Lemma 3.4 that this solution is always bounded.

Theorem 4.5. In the case $r>p$, if $J^{*}\left(v_{0}\right) \leq 0$, then (3.1) has a positive solution for $n \geq 0$ at least until the time $T_{1}$ defined in (2.6).

If $J^{*}\left(v_{0}\right)>0,(3.1)$ has a positive solution during a nonempty lapse of time; this solution may become null only after the time $T_{e_{1}}$, or blow up only after the time $T_{1}$.

Proof. In this case, from Lemma 3.4, (3.1) has a bounded solution at least until the time $T_{1}$ and from the inequality (4.3), we get

$$
\begin{aligned}
\left\|v_{n}\right\|_{p+1}^{p+1}-\left\|v_{n+1}\right\|_{p+1}^{p+1} & \\
\leq & \frac{p+1}{p} \Delta t\left(J^{*}\left(v_{n}\right)-\left(\alpha_{1} \frac{r-1}{r+1}+\alpha_{2}\left(\frac{r-1}{r+1}-\frac{p-1}{p+1}\right)\right) \int_{\Omega} v_{n}^{r+1} d x\right) \\
& \quad-\alpha_{2} \frac{p-1}{p} \Delta t \int_{\Omega} v_{n}^{r-p} v_{n+1}^{p+1} d x,
\end{aligned}
$$

and again we obtain

$$
\left\|v_{n}\right\|_{p+1}^{p+1}-\left\|v_{n+1}\right\|_{p+1}^{p+1} \leq 2 \frac{p+1}{p} \Delta t J^{*}\left(v_{n}\right) .
$$

Then the inequality (4.7) holds and we conclude as in the previous theorem.

Theorem 4.6. If $r \leq p$ and $J^{*}\left(v_{0}\right) \leq 0$, then $\lim _{n \longrightarrow+\infty}\left\|v_{n}\right\|_{p+1}=+\infty$, (except in the case $r=1$ and $\left.J^{*}\left(v_{0}\right)=0\right)$.

The proof of this theorem is analogous to the proof of Theorem 2.4.

Theorem 4.7. If $r>p$ and $J^{*}\left(v_{0}\right) \leq 0$, then the solution of the numerical scheme blows up in a finite time $T_{b}^{*}$ such that

$$
T_{1} \leq T_{b}^{*} \leq T_{2}^{\prime} \text { with } T_{2}^{\prime} \geq T_{2} \text { defined in (2.15). }
$$

Proof. According to the definition of $J_{n}$ in Lemma 3.3, we have

$$
\frac{p-1}{p} \Delta t J_{n}\left(v_{n}\right)=-\left\|v_{n}\right\|_{p+1}^{p+1}+\frac{p-1}{p} \Delta t\left(2 J^{*}\left(v_{n}\right)+\left(\alpha_{2}-\frac{r-1}{r+1} \alpha\right)\left\|v_{n}\right\|_{r+1}^{r+1}\right) .
$$

Since the sequence $\left(J^{*}\left(v_{n}\right)\right)_{n \geq 0}$ is decreasing, if $J^{*}\left(v_{0}\right) \leq 0$, then we get $J^{*}\left(v_{n}\right) \leq 0$; hence

$$
\left\|v_{n}\right\|_{p+1}^{p+1}+\left(\frac{r-1}{r+1} \alpha-\alpha_{2}\right) \frac{p-1}{p} \Delta t\left\|v_{n}\right\|_{r+1}^{r+1} \leq-\frac{p-1}{p} \Delta t J_{n}\left(v_{n}\right) .
$$


From the inequality (4.4) we have

$$
-\frac{p-1}{p} \Delta t J_{n}\left(v_{n}\right) \leq\left\|v_{n+1}\right\|_{p+1}^{p-1}\left(\int_{\Omega}\left(1-\alpha_{2} \frac{p-1}{p} \Delta t v_{n}^{r-p}\right) v_{n}^{p+1} d x\right)^{\frac{2}{p+1}} .
$$

Therefore, by using this estimate in (4.9), we obtain

$$
\begin{aligned}
& \left\|v_{n}\right\|_{p+1}^{p+1}+\left(\frac{r-1}{r+1} \alpha-\alpha_{2}\right) \frac{p-1}{p} \Delta t\left\|v_{n}\right\|_{r+1}^{r+1} \\
& \quad \leq\left\|v_{n+1}\right\|_{p+1}^{p-1}\left(\left\|v_{n}\right\|_{p+1}^{p+1}-\alpha_{2} \frac{p-1}{p} \Delta t\left\|v_{n}\right\|_{r+1}^{r+1}\right)^{\frac{2}{p+1}}
\end{aligned}
$$

hence we get

$$
\left\|v_{n}\right\|_{p+1}^{p-1} \frac{1-\left(\frac{r-1}{r+1} \alpha-\alpha_{2}\right) \frac{p-1}{p} \Delta t \frac{\left\|v_{n}\right\|_{r+1}^{r+1}}{\left\|v_{n}\right\|_{p+1}^{p+1}}}{\left(1-\alpha_{2} \frac{p-1}{p} \Delta t \frac{\left\|v_{n}\right\|_{r+1}^{r+1}}{\left\|v_{n}\right\|_{p+1}^{p+1}}\right)^{\frac{2}{p+1}}} \leq\left\|v_{n+1}\right\|_{p+1}^{p-1} .
$$

Besides, by taking into account the inequality $(1-q x)^{-1} \leq(1-x)^{-q}(0 \leq q \leq 1$, $0 \leq x \leq 1)$ and using a Holder inequality, we deduce that

$$
\left\|v_{n}\right\|_{p+1}^{p-1} \frac{1+\left(\frac{r-1}{r+1} \alpha-\alpha_{2}\right) \frac{p-1}{p} \Delta t C_{2}(\Omega)\left\|v_{n}\right\|_{p+1}^{r-p}}{1-\frac{2}{p+1} \alpha_{2} \frac{p-1}{p} \Delta t C_{2}(\Omega)\left\|v_{n}\right\|_{p+1}^{r-p}} \leq\left\|v_{n+1}\right\|_{p+1}^{p-1} .
$$

In order to simplify the notations, let us denote

$$
\begin{gathered}
\gamma=\frac{2}{p+1} \frac{p-1}{p} \alpha_{2} C_{2}(\Omega), \\
\beta=\left(\frac{r-1}{r+1} \alpha-\alpha_{2}\right) C_{2}(\Omega) \frac{p-1}{p}, \\
\delta=C_{2}(\Omega) \frac{p-1}{p}, z_{n}=\left\|v_{n}\right\|_{p+1} .
\end{gathered}
$$

It is easy to verify that the quantity $(\gamma+\beta)$ is positive. From (4.11), the sequence $\left(z_{n}\right)_{n \geq 0}$ is increasing and satisfies

$$
(\gamma+\beta) \Delta t \frac{z_{n}^{r-1}}{1-\gamma \Delta t z_{n}^{r-p}} \leq z_{n+1}^{p-1}-z_{n}^{p-1} .
$$

Otherwise, for $a, b>0$, we have the inequality (see [14])

$$
b^{p-1}-a^{p-1} \leq \frac{p-1}{r-p} b^{r-1}\left(a^{p-r}-b^{p-r}\right) .
$$

Hence we obtain

$$
\frac{r-p}{p-1}(\gamma+\beta) \Delta t \frac{z_{n}^{r-1} z_{n+1}^{1-r}}{1-\gamma \Delta t z_{n}^{r-p}} \leq z_{n}^{p-r}-z_{n+1}^{p-r} ;
$$

namely,

$$
\gamma \Delta t+\left((\gamma+\beta) \frac{r-p}{p-1}\left(\frac{z_{n}}{z_{n+1}}\right)^{r-1}-\gamma\left(\frac{z_{n}}{z_{n+1}}\right)^{r-p}\right) \Delta t \leq z_{n}^{p-r}-z_{n+1}^{p-r} .
$$


Let us consider the function $f: x \longmapsto(\gamma+\beta) \frac{r-p}{p-1} x^{r-1}-\gamma x^{r-p}$. The previous inequality is written

$$
\gamma \Delta t+f\left(\frac{z_{n}}{z_{n+1}}\right) \Delta t \leq z_{n}^{p-r}-z_{n+1}^{p-r} .
$$

The function $f$ achieves its minimum at the point $x_{0}$ such that $x_{0}^{p-1}=\frac{p-1}{r-1} \frac{\gamma}{\gamma+\beta}$ and is decreasing on the interval $\left[0, x_{0}\right]$, then we shall use the estimates, $f\left(\frac{z_{n}}{z_{n+1}}\right) \geq f(1)$ if $x_{0} \geq 1$, and $f\left(\frac{z_{n}}{z_{n+1}}\right) \geq f\left(x_{0}\right)$ if $x_{0}<1$.

If $x_{0}<1$, we obtain $\gamma \frac{r-p}{r-1} \Delta t \leq z_{n}^{p-r}-z_{n+1}^{p-r} ;$ if $x_{0} \geq 1$, we get $(\gamma+\beta) \frac{r-p}{p-1} \Delta t \leq$ $z_{n}^{p-r}-z_{n+1}^{p-r}$.

It remains to know the sign of $\left(x_{0}-1\right)$ according to $r$ and $p$. Let $r_{0} \in[p, 2 p-1]$ be the unique root of the equation $\left(r_{0}+1\right)^{3}=2(p+1)\left(r_{0}^{2}+1\right)$. We easily get the following results.

- If $p \leq r \leq r_{0}$, then $x_{0}<1$ and we obtain

$$
\left\|v_{n}\right\|_{p+1}^{r-p} \geq\left\|v_{0}\right\|_{p+1}^{r-p}\left(\frac{T_{2}^{\prime}}{T_{2}^{\prime}-t_{n}}\right)
$$

with $T_{2}^{\prime}=\frac{(p+1)(r-1)^{2}}{2(r-p)(r+1)} T_{2}$; we conclude as in Theorem 2.4.

- If $r_{0}<r \leq 2 p-1$, then $x_{0}>1$ and the inequality (4.14) holds with $T_{2}^{\prime}=$ $\frac{(r-1)(p+1)}{p^{2}-1-(r-p)^{2}} T_{2}$.

- If $2 p-1<r \leq p+\sqrt{p^{2}-1}$, then $x_{0} \geq 1$ and (4.14) holds with $T_{2}^{\prime}=$ $\frac{(r-1)(p+1)}{2(r-p)} T_{2}$.

- If $r \geq p+\sqrt{p^{2}-1}$, then $x_{0}<1$ and (4.14) holds with $T_{2}^{\prime}=\frac{(r-1)^{2}(p+1)}{2(r+1)(p-1)} T_{2}$.

Thus we obtain an upper bound on the blow up time according the values of $r$ and $p$ and we can check that $T_{2}^{\prime} \geq T_{2}$ in all cases.

Remark 4.8. The difference between the times $T_{2}$ and $T_{2}^{\prime}$ proceeds from the upper bound of the second member of the inequality (4.4) we used to obtain (4.10).

We now set up a sufficient condition on the initial data for the numerical solution to vanish in a finite time.

Theorem 4.9. If $r \leq p$ and if $v_{0}$ satisfies (2.20), then the solution of (3.1) vanishes in a finite time $T_{e}^{*}$ such that $T_{e_{1}} \leq T_{e}^{*} \leq T_{e_{2}}$ with $T_{e_{2}}$ defined in (2.22).

The proof of this theorem is analogous to that of Theorem 2.5.

Theorem 4.10. If $p<r<2 p-1$ and if $v_{0}$ satisfies

$$
\alpha \frac{r-1}{p-1}\left\|v_{0}\right\|_{p+1}^{p-1}\left\|v_{0}\right\|_{\infty}^{r-p} \leq\left(1-\alpha \frac{p-1}{p}\left\|v_{0}\right\|_{\infty}^{r-p} \Delta t\right) C(\Omega),
$$

then the solution of (3.1) vanishes in a finite time $T_{e}^{*}$ such that $T_{e_{1}} \leq T_{e}^{*} \leq T_{e_{3}}^{\prime}$. $A n d$, if there exists a positive constant $\delta_{1}$ such that

$$
\alpha \frac{r-1}{p-1}\left\|v_{0}\right\|_{\infty}^{r-p}\left\|v_{0}\right\|_{p+1}^{p-1} \leq C(\Omega)\left(1-\delta_{1}\right),
$$

then we have the estimate $T_{e_{3}}-T_{e_{3}}^{\prime}=O(\Delta t)$ with $T_{e_{3}}$ defined in (2.27). 
Proof. The inequality (4.1) may be written as

$$
\begin{aligned}
\left\|v_{n+1}\right\|_{p+1}^{p-1}-\left\|v_{n}\right\|_{p+1}^{p-1} & \\
\leq & \frac{p-1}{p} \Delta t \frac{1}{\left\|v_{n+1}\right\|_{p+1}^{2}} \\
& \times\left(-\int_{\Omega}\left|\nabla v_{n+1}\right|^{2} d x+\alpha_{2} \int_{\Omega} v_{n}^{r-p} v_{n+1}^{p+1} d x+\alpha_{1} \int_{\Omega} v_{n}^{r-1} v_{n+1}^{2} d x\right)
\end{aligned}
$$

and by using the Sobolev constant $C(\Omega)$ we easily get

$$
\begin{aligned}
& \left\|v_{n+1}\right\|_{p+1}^{p-1}-\left\|v_{n}\right\|_{p+1}^{p-1} \\
& \quad \leq \Delta t \frac{p-1}{p}\left(-C(\Omega)+\alpha_{2}\left\|v_{n}\right\|_{\infty}^{r-p}\left\|v_{n+1}\right\|_{p+1}^{p-1}+\alpha_{1}\left\|v_{n}\right\|_{\infty}^{r-p}\left\|v_{n+1}\right\|_{p+1}^{p-1}\right) ;
\end{aligned}
$$

namely

$$
\begin{aligned}
& \left\|v_{n+1}\right\|_{p+1}^{p-1}\left(1-\alpha_{2} \frac{p-1}{p} \Delta t\left\|v_{n}\right\|_{\infty}^{r-p}\right) \\
& \quad \leq\left\|v_{n}\right\|_{p+1}^{p-1}\left(1+\alpha_{1} \frac{p-1}{p} \Delta t\left\|v_{n}\right\|_{\infty}^{r-p}\right)-\frac{p-1}{p} \Delta t C(\Omega) .
\end{aligned}
$$

From Lemma 3.4, we have $\left\|v_{n}\right\|_{\infty}^{r-p} \leq \frac{T_{1}}{T_{1}-t_{n}}\left\|v_{0}\right\|_{\infty}^{r-p}$; that is, $\left\|v_{n}\right\|_{\infty}^{r-p} \leq \frac{p}{r-p} \frac{1}{\alpha} \frac{1}{T_{1}-t_{n}}$.

Hence we obtain

$$
\begin{aligned}
& \left\|v_{n+1}\right\|_{p+1}^{p-1}\left(1-\frac{\alpha_{2}}{\alpha} \frac{p-1}{r-p} \Delta t \frac{1}{T_{1}-t_{n}}\right) \\
& \quad \leq\left\|v_{n}\right\|_{p+1}^{p-1}\left(1+\frac{\alpha_{1}}{\alpha} \frac{p-1}{r-p} \Delta t \frac{1}{T_{1}-t_{n}}\right)-\frac{p-1}{p} \Delta t C(\Omega) .
\end{aligned}
$$

By using the notations $C_{1}=\frac{p-1}{p} C(\Omega)$ and $\mu=\frac{p-1}{r-p}$, the previous inequality becomes

$$
\left\|v_{n+1}\right\|_{p+1}^{p-1}\left(T_{1}-t_{n}-\frac{\alpha_{2}}{\alpha} \mu \Delta t\right) \leq\left\|v_{n}\right\|_{p+1}^{p-1}\left(T_{1}-t_{n}+\frac{\alpha_{1}}{\alpha} \mu \Delta t\right)-\Delta t C_{1}\left(T_{1}-t_{n}\right) .
$$

In the case $r<2 p-1$, we have $\frac{\alpha_{2}}{\alpha}=\frac{1}{\mu}$ and $\frac{\alpha_{1}}{\alpha}=\frac{\mu-1}{\mu}$, and by multiplying (4.17) by $\left(T_{1}-t_{n+1}\right)^{\mu-1}$, we obtain

$$
\begin{aligned}
& \left(T_{1}-t_{n+1}\right)^{\mu}\left\|v_{n+1}\right\|_{p+1}^{p-1} \\
& \quad \leq\left\|v_{n}\right\|_{p+1}^{p-1}\left(T_{1}-t_{n}+(\mu-1) \Delta t\right)\left(T_{1}-t_{n+1}\right)^{\mu-1} \\
& \quad-\Delta t C_{1}\left(T_{1}-t_{n}\right)\left(T_{1}-t_{n+1}\right)^{\mu-1} .
\end{aligned}
$$

Since $\mu \geq 1$, we get $\left(T_{1}-t_{n}+(\mu-1) \Delta t\right)\left(T_{1}-t_{n+1}\right)^{\mu-1} \leq\left(T_{1}-t_{n}\right)^{\mu}$ and we obtain

$$
\left\|v_{n+1}\right\|_{p+1}^{p-1}\left(T_{1}-t_{n+1}\right)^{\mu} \leq\left\|v_{n}\right\|_{p+1}^{p-1}\left(T_{1}-t_{n}\right)^{\mu}-C_{1} \Delta t\left(T_{1}-t_{n}\right)\left(T_{1}-t_{n+1}\right)^{\mu-1},
$$

which implies

$$
\left\|v_{n+1}\right\|_{p+1}^{p-1}\left(T_{1}-t_{n+1}\right)^{\mu} \leq\left\|v_{0}\right\|_{p+1}^{p-1} T_{1}^{\mu}-C_{1} \Delta t \sum_{j=0}^{n}\left(T_{1}-t_{j}\right)\left(T_{1}-t_{j+1}\right)^{\mu-1} .
$$

Since the mapping $t \longmapsto\left(T_{1}-t+\Delta t\right)\left(T_{1}-t\right)^{\mu-1}$ is decreasing, we have

$$
\Delta t \sum_{j=0}^{n}\left(T_{1}-t_{j}\right)\left(T_{1}-t_{j+1}\right)^{\mu-1} \geq \int_{\Delta t}^{t_{n+2}}\left(T_{1}-s+\Delta t\right)\left(T_{1}-s\right)^{\mu-1} d s
$$


that is

$$
\begin{aligned}
& \Delta t \sum_{j=0}^{n}\left(T-t_{j}\right)\left(T_{1}-t_{j+1}\right)^{\mu-1} \\
& \quad \geq \frac{1}{\mu+1}\left(\left(T_{1}-\Delta t\right)^{\mu}\left(T_{1}+\frac{\Delta t}{\mu}\right)-\left(T_{1}-t_{n+2}\right)^{\mu}\left(T_{1}-t_{n+1}+\frac{\Delta t}{\mu}\right)\right) .
\end{aligned}
$$

Hence we obtain

$$
\begin{aligned}
\left\|v_{n+1}\right\|_{p+1}^{p-1}\left(T_{1}-t_{n+1}\right)^{\mu} & \\
\leq & \left\|v_{0}\right\|_{p+1}^{p-1}-\frac{C_{1}}{\mu+1}\left(T_{1}-\Delta t\right)^{\mu}\left(T_{1}+\frac{\Delta t}{\mu}\right) \\
& +\frac{C_{1}}{\mu+1}\left(T_{1}-t_{n+2}\right)^{\mu}\left(T_{1}-t_{n+1}+\frac{\Delta t}{\mu}\right) .
\end{aligned}
$$

If $v_{0}$ satisfies

$$
\left\|v_{0}\right\|_{p+1}^{p-1}<\frac{C_{1}}{\mu+1}\left(T_{1}-\Delta t\right)^{\mu}\left(T_{1}+\frac{\Delta t}{\mu}\right),
$$

then the right side of this inequality will be null for $t_{n+1}=T_{e_{3}}^{\prime}$ such that

$$
\begin{aligned}
\left(T_{1}\right. & \left.-T_{e_{3}}^{\prime}+\frac{\Delta t}{\mu}\right)\left(T_{1}-T_{e_{3}}^{\prime}-\Delta t\right)^{\mu} \\
& =\left(T_{1}-\Delta t\right)^{\mu}\left(T_{1}+\frac{\Delta t}{\mu}\right)-\frac{\mu+1}{C_{1}}\left\|v_{0}\right\|_{p+1}^{p-1} T_{1}^{\mu} \\
& =\left(T_{1}-\Delta t\right)^{\mu}\left(T_{1}+\frac{\Delta t}{\mu}\right)-T_{1}^{\mu+1}+\left(T_{1}-T_{e_{3}}\right)^{\mu+1}
\end{aligned}
$$

Since $T_{\mathrm{e}_{3}}^{\prime}$ is bounded, we deduce easily that $\left(T_{1}-T_{\mathrm{e}_{3}}\right)^{\mu+1}-\left(T_{1}-T_{\mathrm{e}_{3}}^{\prime}\right)^{\mu+1}=O(\Delta t)$, which gives $T_{\mathrm{e}_{3}}-T_{\mathrm{e}_{3}}^{\prime}=O(\Delta t)$ if the quantity $\left(T_{1}-T_{\mathrm{e}_{3}}\right)$ is greater than a positive real $\delta$ (independent of $\Delta t$ ). Then, as $\left(T_{1}-T_{\mathrm{e}_{3}}\right)^{\mu+1}=T_{1}^{\mu+1}\left(1-\frac{\mu+1}{C_{1} T_{1}}\right)$, the estimate holds if there exists $\delta_{1}>0$ such that $\frac{\mu+1}{C_{1} T_{1}} \leq 1-\delta_{1}$ or if $v_{0}$ satisfies (4.16).

Besides, as $\left(T_{1}-\Delta t\right)^{\mu}\left(T_{1}+\frac{\Delta t}{\mu}\right) \geq T_{1}^{\mu}\left(T_{1}-\mu \Delta t\right)$, the inequality (4.19) holds if $\left\|v_{0}\right\|_{p+1}^{p-1} \leq \frac{C_{1}}{\mu+1}\left(T_{1}-\mu \Delta t\right) ;$ namely

$$
\alpha \frac{r-1}{p-1}\left\|v_{0}\right\|_{\infty}^{r-p}\left\|v_{0}\right\|_{p+1}^{p-1} \leq\left(1-\alpha \frac{p-1}{p} \Delta t\left\|v_{0}\right\|_{\infty}^{r-p}\right) C(\Omega) .
$$

The lower bound $T_{e_{1}}$ of the extinction time proceeds from Theorem 4.4.

Theorem 4.11. If $r \geq 2 p-1$ and if $v_{0}$ satisfies (2.25), then the solution of the numerical scheme vanishes in a finite time $T_{e}^{*}$ such that $T_{e_{1}} \leq T_{e}^{*} \leq T_{\mathrm{e}_{3}}$.

Proof. In this case, we have $\alpha_{1}=0$ and $\alpha_{2}=\alpha$; then by multiplying the inequality (4.17) by $\left(T_{1}-t_{n}\right)^{\mu-1}$, we obtain

$$
\left\|v_{n+1}\right\|_{p+1}^{p-1}\left(T_{1}-t_{n}-\mu \Delta t\right)\left(T_{1}-t_{n}\right)^{\mu-1} \leq\left\|v_{n}\right\|_{p+1}^{p-1}\left(T_{1}-t_{n}\right)^{\mu}-\Delta t C_{1}\left(T_{1}-t_{n}\right)^{\mu} .
$$

Since the inequality

$$
\left(T_{1}-t_{n}-\mu \Delta t\right)\left(T_{1}-t_{n}\right)^{\mu-1} \geq\left(T_{1}-t_{n+1}\right)^{\mu},
$$

holds, we get

$$
\left\|v_{n+1}\right\|_{p+1}^{p-1}\left(T_{1}-t_{n+1}\right)^{\mu} \leq\left\|v_{n}\right\|_{p+1}^{p-1}\left(T_{1}-t_{n}\right)^{\mu}-C_{1} \Delta t\left(T_{1}-t_{n}\right)^{\mu},
$$


which implies

$$
\left\|v_{n+1}\right\|_{p+1}^{p-1} \leq T_{1}^{\mu}\left\|v_{0}\right\|_{p+1}^{p-1}-C_{1} \Delta t \sum_{j=0}^{n}\left(T_{1}-t_{j}\right)^{\mu}
$$

But,

$$
\Delta t \sum_{j=0}^{n}\left(T_{1}-t_{j}\right)^{\mu} \geq \int_{0}^{t_{n+1}}\left(T_{1}-s\right)^{\mu} d s=\frac{1}{\mu+1}\left(T_{1}^{\mu+1}-\left(T_{1}-t_{n+1}\right)^{\mu+1}\right) .
$$

From this estimate and (4.20), we obtain

$$
\left\|v_{n}\right\|_{p+1}^{p-1} \leq\left(\left\|v_{0}\right\|_{p+1}^{p-1}-\frac{C_{1} T_{1}}{\mu+1}\right) \frac{T_{1}^{\mu}}{\left(T_{1}-t_{n}\right)^{\mu}}+\frac{C_{1}}{\mu+1}\left(T_{1}-t_{n}\right) .
$$

If $\left\|v_{0}\right\|_{p+1}^{p-1} \leq \frac{C_{1} T_{1}}{\mu+1}$, then the right side of this inequality becomes null at $t_{n}=T_{\mathrm{e}_{3}}$. Thus the solution vanishes in a finite time $T_{e}^{*} \leq T_{\mathrm{e}_{3}}$ and from Theorem 4.4 such that $T_{e_{1}} \leq T_{e}^{*}$.

In the particular case $r=1$ we obtain the following results analogous to the theoretical case (see [14]).

- If $\alpha<\lambda_{1}$, the numerical solution vanishes after a time $T_{e}^{*}$ such that

$$
T_{e_{1}} \leq T_{e}^{*} \leq \frac{p}{p-1} \frac{\lambda_{1}}{\lambda_{1}-\alpha} \frac{\left\|v_{0}\right\|_{p+1}^{p-1}}{C(\Omega)} .
$$

- If $\alpha=\lambda_{1}$, the numerical solution converges in $L^{p+1}(\Omega)$ to $\theta \rho_{1}$ with

$$
\theta \rho_{1} \leq\left(\int_{\Omega} v_{0}^{p} \rho_{1} d x\right)^{\frac{1}{p}}
$$

- If $\alpha>\lambda_{1}$, then $\lim _{n \longrightarrow+\infty}\left\|v_{n}\right\|_{p+1}=+\infty$.

In the particular case $r=p$ we have the following inequalities (see [11]):

$$
\frac{p-1}{p} \Delta t G\left(v_{n+1}\right) \leq\left(1+\alpha \frac{p-1}{p} \Delta t\right)\left\|v_{n}\right\|_{p+1}^{p-1}-\left\|v_{n+1}\right\|_{p+1}^{p-1} \leq \frac{p-1}{p} \Delta t G\left(v_{n}\right) .
$$

Since the sequence $\left(G\left(v_{n}\right)\right)_{n \geq 0}$ is decreasing, we obtain

$$
\left\|v_{n}\right\|_{p+1}^{p-1} \geq\left(1+\alpha \frac{p-1}{p} \Delta t\right)^{n}\left(\left\|v_{0}\right\|_{p+1}^{p-1}-\frac{G\left(v_{0}\right)}{\alpha}\right)+\frac{G\left(v_{0}\right)}{\alpha}
$$

and

$$
\left\|v_{n}\right\|_{p+1}^{p-1} \leq\left(1+\alpha \frac{p-1}{p} \Delta t\right)^{n}\left(\left\|v_{0}\right\|_{p+1}^{p-1}-\frac{G\left(v_{n}\right)}{\alpha}\right)+\frac{G\left(v_{n}\right)}{\alpha} .
$$

Therefore, if $v_{0}$ satisfies $\alpha\left\|v_{0}\right\|_{p+1}^{p-1}<C(\Omega)$, there exists a time $T_{e}=N \Delta t$ such that the solution of the numerical scheme becomes null and we have

$$
\left(1+\alpha \frac{p-1}{p} \Delta t\right)^{N} \leq \frac{C(\Omega)}{C(\Omega)-\alpha\left\|v_{0}\right\|_{p+1}^{p-1}} .
$$

- If $v_{0}$ satisfies $\alpha\left\|v_{0}\right\|_{p+1}^{p-1}>G\left(v_{0}\right)$, then $\lim _{n \longrightarrow+\infty}\left\|v_{n}\right\|_{p+1}=+\infty$. 


\section{CONVERGEnCE OF THE NUMERICAL SCHEME}

Let $T_{\Delta t}^{*}$ be the time existence of the numerical solution. We define a piecewise linear approximation $u_{\Delta t}$ of the weak solution $u$ of (1.1) by

$$
u_{\Delta t=v_{n}^{p}}+\frac{t-t_{n}}{\Delta t}\left(v_{n+1}^{p}-v_{n}^{p}\right) \quad \forall t \in\left[t_{n}, t_{n+1}\right], \quad t \leq T_{\Delta T}^{*},
$$

and we denote $T^{*}=\inf _{0<\Delta t<\Delta t_{0}} T_{\Delta t}^{*}\left(T^{*} \geq T_{1}\right)$.

Let $T<T^{*}$. Here we prove that the solution of the numerical scheme converges to the weak solution of (1.1) on the interval $[0, T]$.

We need the following two lemmas.

Lemma 5.1. If $v_{0} \in H_{0}^{1}(\Omega) \cap C^{\epsilon}(\bar{\Omega}),(\epsilon \in] 0,1[)$, then there exists a positive constant $C$ depending on $\Omega, \alpha, p, r$ such that

$$
\sum_{n=0}^{N} \int_{\Omega} v_{n+1}\left(v_{n+1}-v_{n}\right)\left(v_{n+1}^{p-1}-v_{n}^{p-1}\right) d x \leq \frac{p-1}{p} \Delta t(1+C \Delta t)\left(J^{*}\left(v_{0}\right)-J^{*}\left(v_{N}\right)\right) .
$$

Proof. By multiplying (3.1) by $\left(v_{n+1}-v_{n}\right)$ and integrating on $\Omega$ we get

$$
\begin{aligned}
& \frac{p}{(p-1) \Delta t} \int_{\Omega} v_{n+1}\left(v_{n+1}-v_{n}\right)\left(v_{n+1}^{p-1}-v_{n}^{p-1}\right) d x \\
& =\left[-\frac{1}{2} \int_{\Omega}\left|\nabla v_{n+1}\right|^{2} d x+\frac{1}{2} \int_{\Omega}\left|\nabla v_{n}\right|^{2} d x+\alpha \int_{\Omega} v_{n}^{r}\left(v_{n+1}-v_{n}\right) d x\right] \\
& +\left[-\frac{1}{2} \int_{\Omega}\left|\nabla\left(v_{n+1}-v_{n}\right)\right|^{2} d x\right. \\
& \quad+\alpha_{2} \int_{\Omega} v_{n}^{r-p}\left(v_{n+1}-v_{n}\right)\left(v_{n+1}^{p}-v_{n}^{p}\right) d x \\
& \left.\quad+\alpha_{1} \int_{\Omega} v_{n}^{r-1}\left(v_{n+1}-v_{n}\right)^{2} d x\right] .
\end{aligned}
$$

By using the Young inequality, we have

$$
\alpha \int_{\Omega} v_{n}^{r}\left(v_{n+1}-v_{n}\right) d x \leq \frac{\alpha}{r+1}\left(\left\|v_{n+1}\right\|_{r+1}^{r+1}-\left\|v_{n}\right\|_{r+1}^{r+1}\right)
$$

hence

$$
-\frac{1}{2} \int_{\Omega}\left|\nabla v_{n+1}\right|^{2} d x+\frac{1}{2} \int_{\Omega}\left|\nabla v_{n}\right|^{2} d x+\alpha \int_{\Omega} v_{n}^{r}\left(v_{n+1}-v_{n}\right) d x \leq J^{*}\left(v_{n}\right)-J^{*}\left(v_{n+1}\right) .
$$

Besides, we have

$$
-\frac{1}{2} \int_{\Omega}\left|\nabla\left(v_{n+1}-v_{n}\right)\right|^{2} d x \leq-\frac{\lambda_{1}}{2} \int_{\Omega}\left(v_{n+1}-v_{n}\right)^{2} d x .
$$

If $r \leq p$, then $\alpha_{1}=\alpha$ and $\alpha_{2}=0$ and the second part of the second member of (5.3) may be bounded by

$$
\int_{\Omega}\left(\alpha v_{n}^{r-1}-\frac{\lambda_{1}}{2}\right)\left(v_{n+1}-v_{n}\right)^{2} d x .
$$


Let us denote $\Omega_{+}=\left\{x \in \Omega / v_{n}^{r-1}(x)>\frac{\lambda_{1}}{2 \alpha}\right\}$; so the quantity (5.6) may be bounded by

$$
\int_{\Omega_{+}} \alpha v_{n}^{r-1}\left(v_{n+1}-v_{n}\right)^{2} d x \leq \alpha\left(\frac{2 \alpha}{\lambda_{1}}\right)^{\frac{p-r}{r-1}} \int_{\Omega} v_{n}^{p-1}\left(v_{n+1}-v_{n}\right)^{2} d x,
$$

and from Lemma 4.3, we obtain

$$
\int_{\Omega}\left[\alpha v_{n}^{r-1}-\frac{\lambda_{1}}{2}\right]\left(v_{n+1}-v_{n}\right)^{2} d x \leq C \Delta t\left(J^{*}\left(v_{n}\right)-J^{*}\left(v_{n+1}\right)\right)
$$

with $C=2 \alpha \frac{p+1}{p}\left(\frac{2 \alpha}{\lambda_{1}}\right)^{\frac{p-r}{r-1}}$.

If $r>p$, then we denote $M=\sup _{n \Delta t \leq T}\left\|v_{n}\right\|_{\infty}$, and the second part of the second member of (5.3) is bounded by

$$
\int_{\Omega}\left(\alpha_{1} M^{r-p} v_{n}^{p-1}+\alpha_{2} p M^{p-1} v_{n}^{r-p}-\frac{\lambda_{1}}{2}\right)\left(v_{n+1}-v_{n}\right)^{2} d x .
$$

If we denote $\Omega_{+}=\left\{x \in \Omega / \alpha_{2} p M^{p-1} v_{n}^{r-p}>\frac{\lambda_{1}}{2}\right\}$, then this quantity is bounded by

$$
\int_{\Omega_{+}}\left(\alpha_{1} M^{r-p} v_{n}^{p-1}+\alpha_{2} p M^{p-1} v_{n}^{r-p}\right)\left(v_{n+1}-v_{n}\right)^{2} d x .
$$

If $r<2 p-1$, then we have the estimate $v_{n}^{r-p} \leq\left(\frac{2 \alpha_{2} p M^{p-1}}{\lambda_{1}}\right)^{\frac{2 p-r-1}{r-p}} v_{n}^{p-1}$ on $\Omega_{+}$, and if $r \geq 2 p-1$, then we have the estimate $v_{n}^{r-p} \leq M^{r-2 p-1} v_{n}^{p-1}$. So in these two cases, we obtain

$$
\begin{aligned}
\int_{\Omega}\left(\alpha_{1} M^{r-p} v_{n}^{r-p}+\alpha_{2} p M^{p-1} v_{n}^{r-p}-\frac{\lambda_{1}}{2}\right) & \left(v_{n+1}-v_{n}\right)^{2} d x \\
& \leq C_{1} \int_{\Omega} v_{n}^{p-1}\left(v_{n+1}-v_{n}\right)^{2} d x
\end{aligned}
$$

with $C_{1}=\operatorname{Max}\left(\alpha_{1} M^{r-p}+\alpha_{2} p M^{p-1}\left(\frac{2 \alpha_{2} p M^{p-1}}{\lambda_{1}}\right)^{\frac{2 p-r-1}{r-p}}, \alpha_{1} M^{r-p}+\alpha_{2} p M^{r-p}\right)$.

By using Lemma 4.3 we deduce that

$$
\begin{aligned}
\int_{\Omega}\left(\alpha_{1} M^{r-p} v_{n}^{r-p}+\alpha_{2} p M^{p-1} v_{n}^{r-p}\right. & \left.-\frac{\lambda_{1}}{2}\right)\left(v_{n+1}-v_{n}\right)^{2} d x \\
& \leq 2 \frac{p+1}{p} C_{1} \Delta t\left(J^{*}\left(v_{n}\right)-J^{*}\left(v_{n+1}\right)\right) .
\end{aligned}
$$

Then from the inequalities (5.3), (5.4), (5.7), (5.9), we obtain the lemma.

Lemma 5.2. If $N \Delta t \leq T$, then there exists a positive constant $C(p)$ independent of the initial condition such that

$$
\begin{aligned}
& \sum_{n=0}^{N} \int_{\Omega} \mid v_{n+1}^{p}-v_{n}^{p}-\frac{p}{p-1} v_{n+1}\left(v_{n+1}^{p-1}-v_{n}^{p-1}\right) \mid d x \\
& \leq C \Delta t^{\frac{p-1}{p+1}}\left(J^{*}\left(v_{0}\right)-J^{*}\left(v_{N}\right)\right)^{\frac{p}{p+1}} .
\end{aligned}
$$


Proof. We recall a result shown in [10]: there a positive constant $C(p)$ such that

$$
\begin{gathered}
\sum_{n=0}^{N} \int_{\Omega}\left|v_{n+1}^{p}-v_{n}^{p}-\frac{p}{p-1} v_{n+1}\left(v_{n+1}^{p-1}-v_{n}^{p-1}\right)\right| d x \\
\leq C(p) N^{\frac{1}{p+1}}\left[\left(\sum_{n=0}^{N} \int_{\Omega} v_{n+1}\left(v_{n+1}-v_{n}\right)\left(v_{n+1}^{p-1}-v_{n}^{p-1}\right) d x\right)^{\frac{p}{p+1}}\right. \\
\left.+\left(\sum_{n=0}^{N} \int_{\Omega} v_{n}^{p-1}\left(v_{n+1}-v_{n}\right)^{2} d x\right)^{\frac{p}{p+1}}\right] .
\end{gathered}
$$

With Lemmas 5.1 and 4.3, the right side of this inequality is bounded by

$$
\begin{aligned}
C(p) N^{\frac{1}{p+1}}\left[\left(\frac{p-1}{p} \Delta t\right.\right. & \left.(1+C \Delta t)\left(J^{*}\left(v_{0}\right)-J^{*}\left(v_{N}\right)\right)\right)^{\frac{p}{p+1}} \\
+ & \left.\left(2 \frac{p+1}{p} \Delta t\left(J^{*}\left(v_{0}\right)-J^{*}\left(v_{N}\right)\right)\right)^{\frac{p}{p+1}}\right]
\end{aligned}
$$

so, for $N \Delta t \leq T$, by $C \Delta t^{\frac{p-1}{p+1}}\left(J^{*}\left(v_{0}\right)-J^{*}\left(v_{N}\right)\right)^{\frac{p}{p+1}}$.

Theorem 5.3. The function $u_{\Delta t}$ converges when $\Delta t$ tends to 0 , to the weak solution of problem (1.1) in $C\left(0, T ; L^{1}(\Omega)\right)$.

Proof. The function $u_{\Delta t}$ is bounded in $C\left(0, T ; L^{\infty}(\Omega)\right)$ from Lemma 3.4 and in $C\left(0, T ; H_{0}^{1}(\Omega)\right)$. Besides, in the same manner as in [10], we obtain that $\frac{d}{d t} u_{\Delta t} \in$ $L^{2}\left(0, T ; L^{1}(\Omega)\right)$. Hence, there exists a subsequence, again labeled $u_{\Delta t}$, such that $u_{\Delta t}$ converges to some function $u$ in $C\left(0, T ; L^{q}(\Omega)\right)$ with $q<\frac{2 d}{d-2}$ if $d>2, q<\infty$ if $d=2$ and in $C(0, T ; \Omega)$ if $d=1$ ([19]).

It remains to prove that $u$ is a weak solution of problem (1.1). Let $\varphi$ be a test function in $C^{2}(\Omega \times(0, T)) \cap C^{1}(\bar{\Omega} \times[0, T]) ; \varphi(x, t)=0$ for $x \in \partial \Omega$.

Multiplying the equality (3.1) by $\varphi$ and integrating on $\Omega$, we get

$$
\begin{aligned}
\int_{\Omega} \frac{p}{p-1} v_{n+1} & \left(v_{n+1}^{p-1}-v_{n}^{p-1}\right) \varphi d x \\
& +\Delta t \int_{\Omega}\left(v_{n+1} A \varphi-\alpha_{1} v_{n+1} v_{n}^{r-1} \varphi-\alpha_{2} v_{n+1}^{p} v_{n}^{r-1} \varphi\right) d x=0 ;
\end{aligned}
$$

hence, for $T=N \Delta t$ we get

$$
\begin{aligned}
& \sum_{n=0}^{N-1} \frac{1}{\Delta t} \int_{t_{n}}^{t_{n+1}} \int_{\Omega} \frac{p}{p-1} v_{n+1}\left(v_{n+1}^{p-1}-v_{n}^{p-1}\right) \varphi d x d t \\
& \quad+\sum_{n=0}^{N-1} \int_{t_{n}}^{t_{n+1}} \int_{\Omega}\left(v_{n+1} A \varphi-\alpha_{1} v_{n+1} v_{n}^{r-1} \varphi-\alpha_{2} v_{n+1}^{p} v_{n}^{r-1} \varphi\right) d x d t=0 .
\end{aligned}
$$


The first term of this equality may be written as

$$
\begin{aligned}
\sum_{n=0}^{N-1} \frac{1}{\Delta t} \int_{t_{n}}^{t_{n+1}} \int_{\Omega}\left(\frac{p}{p-1} v_{n+1}\left(v_{n+1}^{p-1}-v_{n}^{p-1}\right)-v_{n+1}^{p}+v_{n}^{p}\right) & \varphi d x d t \\
& +\int_{0}^{T} \int_{\Omega} \frac{d}{d t} u_{\Delta t} \varphi d x d t .
\end{aligned}
$$

From (5.10) the first part of this equality tends to zero when $\Delta t \longrightarrow 0$ and the second part tends to

$$
-\int_{0}^{T} \int_{\Omega} u \frac{d \varphi}{d t} d x d t+\int_{\Omega} u(x, T) \varphi(x, T) d x-\int_{\Omega} u_{0}(x) \varphi(x, 0) d x .
$$

We prove in a classical manner ([14]) that the second term of (5.11) when $\Delta t \longrightarrow 0$ tends to

$$
\int_{0}^{T} \int_{\Omega}\left(u^{\frac{1}{p}} A \varphi-\alpha u^{\frac{r}{p}} \varphi\right) d x d t
$$

So $u$ satisfies the equation

$$
\begin{aligned}
-\int_{0}^{T} \int_{\Omega} u \frac{d \varphi}{d t} d x d t & +\int_{0}^{T} \int_{\Omega}\left(u^{\frac{1}{p}} A \varphi-\alpha u^{\frac{r}{p}} \varphi\right) d x d t \\
& =\int_{\Omega} u_{0}(x) \varphi(x, 0) d x-\int_{\Omega} u(x, T) \varphi(x, T) d x
\end{aligned}
$$

and is a weak solution of $(1.1)$

\section{REFERENCES}

[1] H. AMANN, On the existence of positive solutions of nonlinear elliptic boundary value problems, Indiana Univ. Math J., 21 (1971), pp 125-146. MR 45:5558

[2] D. ARONSON, M. CRANDALL, L. PELETIER, Stabilization of solutions of a degenerate nonlinear diffusion problem, Nonlin. Anal., Vol. 6 (1982), pp 1001-1002. MR 84j:35099

[3] J.G. BERRYMAN, C.J. HOLLAND, Stability of the separable solution for fast diffusion, Arch. Rational Mech. Anal. 74 (1980), pp 379-388. MR 81m:35065

[4] A. FRIEDMAN, A.A. LACEY, Blowup of positive solutions of semilinear parabolic equations, Math. Anal. Appl., 132 (1998), pp 171-186.

[5] A. FRIEDMAN, B. MCLEOD, Blowup of positive solutions of semilinear heat equations, Indiana Univ. Math. J. 34, (1985), pp 425-447.

[6] A. FRIEDMAN, B. MCLEOD, Blowup of nonlinear degenarate parabolic equations, Arch. Rational Mech. Anal. 96, (1986), pp 55-80.

[7] H.B. KELLER, Elliptic boundary value problems suggested by nonlinear diffusion process, Arch. Rational Mech. Anal., 96 (1986), pp 55-80.

[8] M.-N. LE ROUX, Semi-discretization in time of nonlinear parabolic equations with blowup of the solution, SIAM J. Numer. Anal. Vol. 31, no. 1, (1994), pp 170-195. MR 95a:65148

[9] M.-N. LE ROUX, Numerical solution of nonlinear reaction diffusion processes in plasmas, Proceedings of the Second Hellenic European Conference on Mathematics and Informatics, Sept. 94, Athenis, E.A. Lipitakis Editor. CMP 96:07

[10] M.-N. LE ROUX, Semidiscretization in time of a fast diffusion equation, J. Math. Anal. Appl. Vol. 137, no. 2, (1989), pp 354-370. MR 90k:65166

[11] M.-N. LE ROUX, Résolution numérique d'un problème de fast-diffusion, Publication CeReMaB, Bordeaux I, No. 9306.

[12] M.-N. LE ROUX, H. WILHELMSSON, External boundary effects on simultaneous diffusion and reaction processes, Physica Scripta, Vol. 40, (1989), pp 674-681.

[13] H.A. LEVINE, P.E. SACKS, Some existence and nonexistence theorems for solutions of degenerate parabolic equations, J. Diff. Equat., Vol. 52, (1984), pp 135-161. MR 85f:35120 
[14] P.E. MAINGE, Résolution numérique d'équations de réaction-diffusion intervenant en physique des plasmas, Thèse (1996), Université Bordeaux 1.

[15] E.S. SABININA, A class of nonlinear degenerating parabolic equations, Soviet Math. Dokl. 143 (1962), pp 495-498.

[16] P.E. SACKS, Continuity of solutions of a singular parabolic equation, Nonlinear Anal., Vol. 7 (1983), pp 387-409. MR 84d:35081

[17] P.E. SACKS, The initial and boundary value problem for a class of degenerate parabolic equations, Part. Diff. Equat., Vol. 8 (1983), pp 693-733. MR 85h:35128

[18] P.E. SACKS, Global behavior for a class of nonlinear evolution equation, SIAM J. Math. Anal. Vol. 16, no. 2, (1985) pp 233-250. MR 85f:35031

[19] J. SIMON, Compact sets in the space $L^{p}(0, T ; B)$, Ann. Mat. Pura., 146 (1987), pp 65-96. MR 89c: 46055

GRAMM-Mathématiques, 351, Cours de la Libération, F-33405 Talence Cedex, France

E-mail address: m.n.leroux@math.u-bordeaux.fr 